\title{
Procedure for High Precision Density Determinations by Hydrostatic Weighing
}

\author{
Horace A. Bowman and Randall M. Schoonover With Appendix by Mildred W. Jones
}

Institute for Basic Standards, National Bureau of Standards, Washington, D.C. 20234

(May 21, 1967)

\begin{abstract}
There are several simplifications which can be made in the performance of the hydrostatic experiment which lead to more reproducible results. The operation of single-pan two-knife balances is discussed, and simple modifications suggested to adapt it to hydrostatic work. A data philosophy is presented which will be particularly applicable to a generation of now-developing force measuring instruments when used in hydrostatic work. A simplified formula for air density is presented and a formula for estimating day-to-day variability in the density of water. A simple balance calibration procedure is presented in an appendix, and simplified methods of fabricating suspension wires and degassing sample surfaces are described. The use of these techniques is illustrated by measurements on silicon crystals which indicate process reproducibility of a standard deviation to about one part per million.
\end{abstract}

Key Words: Air density, balance, balance calibration, balance equilibrium, balance sensitivity, density, double substitution weighing, hydrostatic balance, hydrostatic weighing, silicon density, substitution balance, volumetric measurement, water density, water weighing.

\section{Introduction}

\subsection{Objectives and Accomplishments}

The studies covered by this report were undertaken as a preliminary step to some volumetric and density standardization work being started at NBS $[1]^{1}$ which will involve a great deal of hydrostatic weighing. Primary effort was directed toward a detailed study of the entire hydrostatic weighing process so that major sources of variability could be minimized, and experimental procedures simplified to the extent that nonspecialist workers could perform hydrostatic work approaching the state of the art. Secondary objectives were to evaluate the homogeneity (in density) in silicon boules.

We found, as have others, that major loss of precision occurs due to

(a) Imprecision inherent in the balance used in the hydrostatic work.

(b) Nonreproducibility of the surface tension forces associated with the meniscus formed around the point where the hydrostatic suspension penetrates the airwater interface.

(c) Variability in the buoyant forces exerted on the immersed sample by the gases adhering to its surfaces.

No existing balance was found to be ideal for hydrostatic work. The criteria upon which a balance should be selected are stated and the experiments and tests

${ }^{1}$ Figures in brackets indicate the literature references on page 198. which should be put to any prospective balance are described. Additionally, simple modifications are suggested which will greatly improve balance performance in hydrostatic work without degrading its performance as an ordinary laboratory instrument.

Previous methods of minimizing surface tension errors have involved impractical hit-or-miss techniques, a great deal of luck, critical cleaning processes, detailed optical examination, and a rather small chance of a given suspension wire performing adequately. A simple heating technique was devised which eliminates the necessity for these operations and which gives the experimenter an excellent chance of meniscus stability to 2 or 3 tenths of a dyne per centimeter (a few micrograms in the case of an 0.001 in diam wire) on the "first try."

A simple boiling procedure gives minimization of surface bound gases on test samples at least as well as previous out-gassing methods ordinarily used in this work and with significantly less effort.

A formula is given for estimating air density directly from observations of temperature, pressure, and relative humidity which does not require using tables or graphs. Based upon previous observations of Chappuis and others a formula was constructed for estimating the day-to-day variability in water density.

Finally, we have written a section on "Operating Hints" which contains several items which, although not formally proved, we believe will improve and simplify the overall operation. 
Observations on three silicon crystals from the same boule grown in a vacuum indicated an average density of $2.328932 \mathrm{~g} / \mathrm{cm}^{3}$ with individual crystals spread over a range of $0.000003 \mathrm{~g} / \mathrm{cm}^{3}$. The average density of three crystals cut from a boule grown in argon was $2.329021 \mathrm{~g} / \mathrm{cm}^{3}$ with individual values spread over a range of $0.000002 \mathrm{~g} / \mathrm{cm}^{3}$. Repeat measurements made on all six crystals indicated a process standard deviation of about $1 \mathrm{ppm}$.

We believe that by use of the procedures described in this report, nonspecialist workers can duplicate these results.

\subsection{Historical Background}

There are two methods for determining the density of a solid object: first by independent measurements of its mass and geometry in terms of appropriate standards, from which its density may be calculated; and second, by comparison of its density with some reference density of known or assumed value. Direct measurement is a difficult and time-consuming task, and when great accuracy is called for, it is used only by the most advanced metrology laboratories. The vast majority of density determinations are made by hydrostatic weighing, in which the density of the unknown is obtained by comparison with the (assumed known) density of water.

During the very recent past, several branches of science have called for more precise density measurements. Such data is used in studies of stoichiometry, point defect concentrations in crystals, dimensional stability, atomic constants, and effects of various types of bombardment to name a few. It is well recognized that the larger the sample size, the better will be the precision; but unfortunately, the samples in which there is maximum technical interest are very small indeed. Frequently one needs small samples for verification of homogeneity. Bombarding beams often have limited diameters and penetrating powers, so recent effort has been directed to measurements on small samples.

Hydrostatic measurements on small samples present many problems not present in large sample work. There are three reasons for this:

(a) As balance capacity decreases below $1 \mathrm{~kg}$, percentage precision decreases, although microgram precision increases (but at a lower rate). For example, a modern "convenience-type" balance can compare kilogram masses to $60 \mu \mathrm{g}$ with ease. This is six parts in 100 million. On the other hand, the best 2 -g balance can compare such loads to about $0.5 \mu \mathrm{g}$ or 2.5 parts in 10 million.

(b) A major source of variability in hydrostatic work is meniscus instability. As loads increase, suspension wire size must increase. Tensile capacity increases as cross-section area, but meniscus forces increase only with circumference. Variability in such forces, for various reasons, may increase even more slowly.

(c) A tiny undetected air bubble will cause a much greater loss of precision on a small sample than on a large one.
Present effort to improve small sample work is indicated by two recent reports. In 1964 Henins [2] reported hydrostatic weighings on a group of objects averaging about $4.7 \mathrm{~cm}^{3}$ to a standard deviation of a single observation from the mean of about $4.5 \times 10^{-6}$ $\mathrm{cm}^{3}$. In 1965 Bowman and Schoonover [3], using a cartesian diver technique on a group of $1.2 \mathrm{~cm}^{3}$ objects, reported $1.4 \times 10^{-7} \mathrm{~cm}^{3}$. This is believed the best work to date. The diver is not a practical device for work on samples weighing much more than $10 \mathrm{~g}$ in water. The present studies are concerned with samples weighing from 10 to $20 \mathrm{~g}$ in water.

The foregoing comments have to do with precision and not accuracy. Precision refers to reproducibility of measured value-accuracy to correctness of measured value with respect to some standard. In this report, the measure of precision is the standard deviation of a single independent determination from the mean of several such determinations. In the density area (as in most fields of metrology), precision is better than accuracy which is limited by our imperfect knowledge of the true value of the various reference densities available to us. NBS is aware of its obligations in this area. The present study was undertaken as a preliminary step in the redetermination of several such densities and the development of a family of objects which will collectively become a density standard. However, aside from a few very fundamental studies, most work is aimed at determinations of density changes and density differences, and in work of this type, significant uncertainties in standards can usually be tolerated.

Many experimenters whose experience and training are far removed from metrology are attempting to achieve high quality hydrostatic data with inappropriate apparatus and obsolete techniques. We were particularly impressed by the simplicity of the setup used by Henins, and we used his approach as a starting point. Frequently, after much effort to improve upon it, we returned to it as a "best compromise." We acknowledge the great assistance we received from his work.

\subsection{Hydrostatic Weighing Experiment}

By classical definition, hydrostatic density measurement consists of weighing an object of known mass, $M_{X}$, while it is suspended from a balance in a liquid of some assumed value of density. The indicated loss of mass being equal to the mass of the displaced liquid, we are able to calculate the true volume, $V_{X}$, of the object and (by physical definition of density) calculate the unknown density. Thus

$$
\rho_{X}=\frac{M_{X}}{V_{X}} .
$$

The philosophy proposed here differs slightly from the classical concept. Our experiment consists of measuring the object's apparent weight, $\bar{W}$, in two media of different densities, where apparent weight is defined as the difference between the (downward) gravitational force exerted on the object, and the 
(upward) buoyant force exerted on it by the medium. Thus

$$
\begin{aligned}
& \bar{W}_{A}=g\left(M_{X}-\rho_{A} V_{X}\right) \quad \text { (in air) } \\
& \bar{W}_{W}=g\left(M_{X}-\rho_{W} V_{X}\right) \quad \text { (in water) }
\end{aligned}
$$

A simultaneous solution of the above pair provides values of $M_{X}$ and $V_{X}$ from which density may be calculated.

However, in our experiment, we demand of our balance only that it be linear in force, and the force units may be completely arbitrary -in which case the units for $M_{X}$ and $V_{X}$ will be unrecognizable. But their ratio $\left(M_{X} / V_{X}\right)$ will be in the same units in which $\rho_{W}$ and $\rho_{A}$ are stated, since $\rho_{W}$ and $\rho_{A}$ are the true standards in the density measurement process. At the present state of the art the balance is the most linear force measuring instrument available, and balances (of the type we selected) are calibrated in classical mass units against classical mass standards. However, a new generation of elastic force instruments is growing up, and improving at a faster rate than ordinary balances. When these devices have achieved the precision presently obtainable on balances we believe they will supplant laboratory balances in this application. The verification of force linearity in such devices will be easier than calibration in terms of some particular standard.

As pointed out above, our efforts are primarily directed to improving precision, measured by the reproducibility of independent measurements. The definition of "independent" as used here means an observing process in which all random variables are permitted to operate. This specifically prohibits such procedures as:

(A) Stating a standard deviation in $\bar{W}_{W}$ based upon a series of "immersed pan empty"-"immersed pan loaded" differences, because in this instance the random properties of the meniscus and surface bound gas cannot operate.

(B) Stating a standard deviation in $\bar{W}_{A}$ based upon arresting and releasing the balance with the load stationary on the pan, because a major component of the arrestment error (namely the loading error [4]) cannot operate.

The above restrictions are stated because mistakes of both types have been made and reported in literature. A single independent density determination must consist of the following steps-none of which may be used as a part of a different determination:

(1) A thorough cleaning and degassing of the surfaces of the sample to be tested.

(2) A group of "immersed pan emptv" and "immersed pan loaded" observations, with the differences averaged to provide a single value of $\bar{W}_{W}$.

(3) Removal of the sample from the bath, drying, and temperature soaking followed by a series of air weighings which are averaged to provide a single value of $\bar{W}_{A}$.

In the foregoing procedure, the parameters necessary for the calculation of $\rho_{W}$ are measured in connec- tion with step (2) and those for $\rho_{A}$ in step (3). With measured values of $\bar{W}_{A}$ and $\bar{W}_{W}$, we may solve the simultaneous pair referred to above as follows

$$
\rho_{X}=\frac{\bar{W}_{A} \rho_{W}-\bar{W}_{W} \rho_{A}}{\bar{W}_{A}-\bar{W}_{W}} .
$$

At this point we still have little knowledge upon which to base a precise statement regarding our process reproducibility. To obtain such knowledge, it is necessary to repeat the entire experiment using a new sample of water.

\section{Density of Water}

About 1900 the thermal coefficient of expansion of normal (to be defined) water was measured [5, 6], and in 1937 Tilton and Taylor at NBS [7] published tables of the relative densities of water as a function of temperature from a formula they constructed to fit the early data. This table expresses density in grams per milliliter where the milliliter is defined as the volume occupied by $1 \mathrm{~g}$ of water at $3.98{ }^{\circ} \mathrm{C}$. Around 1910 BIPM $[8,9,10]$ undertook to measure the true volume (in cubic centimeters) of a milliliter by hydrostatically weighing objects of directly measured volume in normal water. These experiments were conducted prior to knowledge of isotopes, and we define "normal" water as that having a "normal" or "average" isotopic abundance ratio. Modern workers [11, 12] have shown that the isotopic abundance ratio in samples of water drawn from various locations on the earth may vary enough to result in density differences of as much as 5 or more parts per million. Without knowledge of the isotopic abundance ratio in the water used in measuring the milliliter we are left with an uncertainty of this amount in the accepted value of the volume of the milliliter. Additionally, there is an even larger source of uncertainty. The vapor pressures of the various isotopic forms of water are significantly different [13], so that distillation to remove impurities or boiling to eliminate dissolved gasses can further upset the isotopic abundance ratio from its "normal" value. Density differences as great as $20 \mathrm{ppm}$ have been found [14] between the first and last fractions of a distillation.

Modern experiments on the thermal dilation of the pure isotope forms and of normal water [15] indicate no serious error in the Tilton and Taylor values; however, there is no modern equivalent of the milliliter evaluation experiment, and it is in this experiment in which significant differences are to be expected. The present work is performed in anticipation of such a repetition. Uncertainty exists in our work associated with the uncertainty in $\rho_{W}$ which cannot be evaluated until this repeat experiment is performed.

Effect of dissolved air. There is very little data available on the effect of dissolved air on the density of water. Figure 1 is a plot of all such data which has come to our attention. The work of Marek is ordinarily used as a basis for making the required corrections, or for 


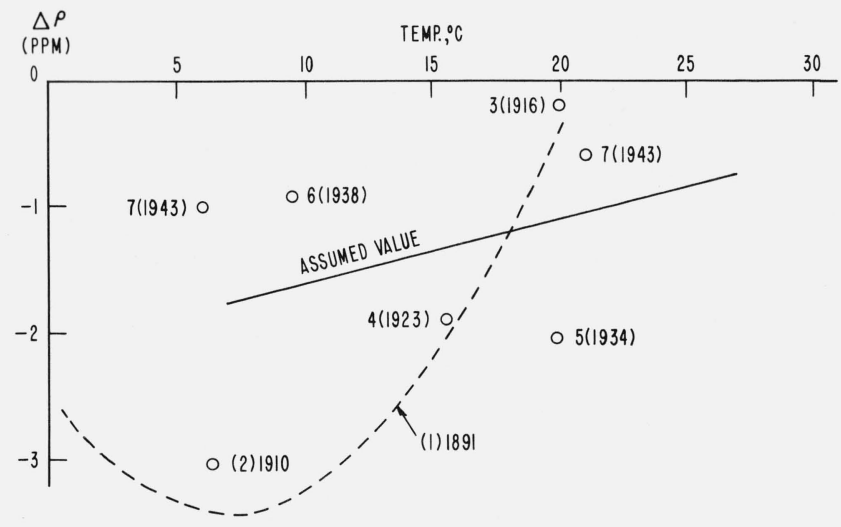

Figure 1. (1) W. Marek, Ann. Physik, 3, 4 (1891). (2) P. Chappusi, Trav. \& Mem. B.I.P.M. 14 (1910). (3) T. W. Richards and Harris, J. Amer. Chem. Soc, 38 (1916). (4) O. E. Frivold, Physik Z. 21, p. 529-534 (1920); 24, p. 86-87 (1923). (5) Emeleus, James, King, J. Chem. Soc, 1207 (1934). (6) Titani and Morita, Bull. Chem. Soc, Japan, 13, 409 (1938). (7) Voskuyl, Davis and Saxer, SAM Report A-727, June (1943).

the justification of ignoring the effect. The plot of his data indicates that extrapolation into the temperature range of ordinary interest would yield extremely improbable values so we reject his data. Dorsey [16] gives other reasons for questioning his work. Our estimate of the probable effect is shown by the solid line whose point slope form is

$$
\Delta \rho_{W}(\text { in ppm })=-2.11+0.053 t_{W}
$$

where $t_{W}$ is water temperature.

Chappuis [17] reported that water reaerates after boiling at a rate such that at $13{ }^{\circ} \mathrm{C}$ and at a depth of $12 \mathrm{~cm}$ below the free surface, 50 percent saturation is achieved after 1 day and 75 percent after 4 days. No other information on the rate of reaeration was located. Our samples are usually held to within a few centimeters of a depth of $12 \mathrm{~cm}$. The correction factor which we apply to the density of water is given by

$$
1-\left(2.11-0.053 t_{W}\right)\left(1-\frac{1}{1+t}\right) \times 10^{-6}
$$

where $t$ is the interval (in days) since deaeration.

Effect of pressure on water density. Water is not incompressible, therefore a correction must be applied to account for the effect of varying pressure. The pressure of the water displaced by the object of interest is a function of the barometric pressure at the free surface and the depth of immersion. The correction factor which we apply is given by

$$
\frac{1}{1-c\left(\frac{B}{760}+\frac{I}{1033}-1\right)}
$$

where $c=$ compressibility $=47.7 \mathrm{ppm} / \mathrm{atm}$

$B=$ barometric pressure (in $\mathrm{mm}$ of $\mathrm{hg}$ )

$I=$ depth of immersion (in $\mathrm{cm}$ ).
The complete formula for $\rho_{W}$ used in our experiments is as follows:

$$
\begin{gathered}
\rho_{W}=\left[1-\frac{\left(t_{W}-3.9863\right)^{2}}{508929.2} \times \frac{t_{W}+288.9414}{t_{W}+68.12963}\right][0.999973] \\
{\left[\frac{1}{1-C\left(\frac{B}{760}+\frac{I}{1033}-1\right)}\right]\left[1-\left(2.11-.053 t_{W}\right)\right.} \\
\left.\left(1-\frac{1}{1+t}\right)\left(10^{-6}\right)\right] .
\end{gathered}
$$

The first term is the Tilton-Taylor formula for $\rho_{W}$ as a function of temperature in grams/milliliter. The second term reduces their values to grams/cubic centimeter. The third term is a correction for pressure and the fourth for dissolved air. The last two terms are ordinarily ignored, however they may alter the calculated value of $\rho_{W}$ by as much as $1.5 \mathrm{ppm}$.

\section{Density of Air}

Major changes in $\rho_{A}$ occur due to variation in the temperature, pressure, and relative humidity, and each of the parameters is carefully observed throughout the experiment. Changes also occur due to variation in the air analysis, however this was not investigated. The instrumentation used for monitoring these ambient parameters is not state-of-the-art-type apparatus, because it is necessary only to assure that the standard deviation in $\rho_{A}$ is held to a few micrograms per cubic centimeter. Apparatus was chosen which is most convenient to use and maintain.

A. Temperature. It will be pointed out in the next section that air density is of interest in two spacesthe weighing chamber of the balance and also in the chamber housing the dial-operated weights. Each of these spaces is monitored by a mercury-in-glass thermometer, divided into $1 / 10^{\circ} \mathrm{C}$ intervals over the range of 20 to $30^{\circ} \mathrm{C}$. The thermometer in the weighing chamber is calibrated for total immersion, and the one which monitors the weight chamber must be satisfied by partial immersion. Inasmuch as a platinum resistance thermometer is used to monitor the hydrostatic bath, these two mercury-in-glass thermometers should be calibrated periodically in this bath.

$B$. Relative humidity is measured on an electric hygrometer of the type discussed by Wexler [18] with its sensing element placed in the weighing chamber of the balance. This instrument usually contains a builtin thermistor type thermometer which is used to monitor the temperature in the immediate vicinity of the r.h. sensing element. This should not be used as a replacement for either of the mercury thermometers. Although it is quite adequate for the use intended by the manufacturer (and it should be used in connection with r.h. meașurements) it will introduce serious uncertainties if used as a replacement for either of the mercury instruments.

Sling and aspiration psychrometers are unsatisfactory in this application. Their required air-flow 
would cause unacceptable disturbances inside a balance, and they lack the required sensitivity and speed of response. Since there are free surfaces of water in the vicinity of the balance in the hydrostatic weighing experiment which exert a strong influence on r.h. the apparatus for monitoring this parameter should be selected with care. Wexler discusses electric hygrometers in detail and we believe that their convenience, speed of response and sensitivity are such that no other type instrument currently available should be considered. Wexler estimates their drift at about 2 percent per year, and feels that semiannual calibration is quite reasonable.

Calibration can be performed by the manufacturer, NBS, or the experimenter himself using various techniques leading to a calibration accuracy of about $1 \frac{1}{2}$ percent using the methods of Wexler and his associates [19].

C. Barometric pressure is most easily observed on an aneroid barometer. Such an instrument should be checked against a mercury barometer. Our barometer is, unfortunately, not equipped with an antiparallax mirror scale. We recommend specifying such a scale for instruments used in this work.

D. The basis for air density calculations. If we have a mass, $m_{G}$, of dry gas at pressure $p$ and absolute temperature $\theta$, whose molecular weight is $M_{G}$, then from the ideal gas law

$$
\begin{gathered}
p V=n R \theta=\left(\frac{m_{G}}{M_{G}}\right) R \theta \\
m_{G}=\frac{p V M_{G}}{R \theta}
\end{gathered}
$$

and

$$
\frac{M_{G}}{R \theta}=\frac{m_{G}}{V} \cdot \frac{1}{p}=\frac{\rho_{G}}{p}
$$

where $n$ is the number of moles involved and $\rho_{G}$ the density of the dry gas.

If we have a mass, $m_{W}$, of water vapor at pressure $e$ and temperature $\theta$ occupying volume $V$, then a similar argument gives

$$
m_{W}=\frac{e V M_{W}}{R \theta}
$$

If the above masses of gas and vapor are mixed in volume $V$, the barometric pressure, $B$, will be (by Dalton's Law of partial pressures) equal to $p+e$ and $p=B-e$. The density, $\rho_{A}$, of the mixture is

$$
\rho_{A}=\frac{m_{G}+m_{W}}{V}=\frac{M_{G}}{R \theta}\left[B-e\left(1-\frac{M_{W}}{M_{G}}\right)\right] .
$$

Using $M_{G} / R \theta$ from eq (2)

$$
\rho_{A}=\frac{\rho_{G}}{P}\left[B-\left(e 1-\frac{M_{W}}{M_{G}}\right)\right] .
$$

If the density of dry gas, $\rho_{G}$, is measured to be $\rho_{0}$ at some reference temperature $\theta_{0}$, and reference pressure $\rho_{0}$, then its density at any temperature $\theta$ and pressure $p$ is

$$
\rho_{G}=\rho_{0}\left(\frac{\theta_{0}}{\theta}\right)\left(\frac{p}{p_{0}}\right)
$$

and using this value of $\rho_{G}$ in the above equation for $\rho_{A}$ gives

$$
\rho_{A}=\left(\frac{\rho_{0} \theta_{0}}{\theta p_{0}}\right)\left[B-e\left(1-\frac{M_{W}}{M_{G}}\right)\right]
$$

On the basis of the natural scale of atomic weights $M_{W}=18.0160$ and $M_{G}=28.966$ [20]. If $\rho_{0}=1.29304$ $\times 10^{-3} \mathrm{~g} / \mathrm{cm}^{3}$ when $\theta_{0}=273.16^{\circ} \mathrm{K}$ and $p_{0}=760 \mathrm{~mm} \mathrm{Hg}$ [21], then

$$
\rho_{A}=0.46475\left(\frac{B-(0.0037803)\left(e_{s}\right)(H)}{t_{A}+273.16}\right)
$$

where $t_{A}$ is temperature in ${ }^{\circ} \mathrm{C}, e_{s}$ the tabulated saturation vapor pressure of water at $t_{A}$ and $H$ the relative humidity expressed as a percentage. This formula assumes moist air behaves in accordance with the ideal gas law, under which circumstances compressibility (defined as $\rho V \mid R \theta$ ) is unity. Goff and Gatch [22] have measured the compressibility of moist air and have found that over the range of temperature, pressure and relative humidity ordinarily existing in the laboratory it varies between 0.9995 and 0.9997 , so the above formula provides values of $\rho_{A}$ too high by this factor. We feel justified in multiplying its right member by an average value of 0.9996 , so that

$$
\rho_{A}=0.46456\left[\frac{B-(0.0037803)\left(e_{s}\right)(H)}{t_{A}+273.16}\right] .
$$

Equation (3) may be used by the experimenter for calculation of air density based upon observed values of temperature, barometric pressure, relative humidity and tabulated values of $e_{s}$, the vapor pressure of water at saturation. In this equation $\rho_{A}$ is a very weak function of $e_{s}$, changing by only $0.24 \times 10^{-6} \mathrm{~g} / \mathrm{cm}^{3}$ for a 1 $\mathrm{mm} \mathrm{Hg}$ change in $e_{s}$. Therefore it is possible to éliminate the inconvenience of using tables by making a very crude approximation for $e_{s}$ in terms of the observed temperature without causing unacceptably large errors in $\rho_{A}$. Most weighings are conducted between 20 and $30{ }^{\circ} \mathrm{C}$, and within this range we may assume

$$
e_{s}=1.435 t_{A}-11.72
$$

under which circumstances

$$
\rho_{A}=\frac{0.464554 B-H\left(0.00252 t_{A}-0.020582\right)}{t_{A}+273.16}
$$

where $\rho_{A}$ is in milligrams per cubic centimeter with adequately small error.

On the rare occasions when it is necessary to perform weighings outside of this temperature range some 
adjustment in the approximation is called for. Over the temperature of 15 to $50{ }^{\circ} \mathrm{C}$, air density may be approximated by

$$
\rho_{A}=\frac{464.56 B-H\left(0.085594 t_{A}^{2}-1.8504 t_{A}+34.47\right)}{t_{A}+273.16}
$$

where $\rho_{A}$ is in micrograms per cubic centimeter. Although these two approximations result in very large errors in $e_{s}$, errors in $\rho_{A}$ associated with their use do not exceed one microgram per cubic centimeter which is quite adequate for weighing work.

In order to test the ability of our system to report small changes in air density reproducibly, it was installed in a balance similar to the one used in the hydrostatic experiments. We made two series of tests (each test over an interval of several days) on the indicated difference in apparent weight of two stainless steel weights of nearly equal mass but of grossly different volume (one weight was hollow). Day-to-day changes in the indicated difference between the apparent weights were taken to be indicative of changes in air density, thus:

$$
\rho_{A}=\frac{\Delta M-\Delta \bar{W}}{\Delta V}
$$

where $\Delta M$ and $\Delta V$ are the mass and volumetric differences between the two weights (measured independently to be $35.466 \mathrm{mg}$ and $42.769 \mathrm{~cm}^{3}$ respectively) and $\Delta \bar{W}$ the difference in apparent weights indicated by the balance. The data is summarized below. Ranges in temperature, barometric pressure and relative humidity, over which observations were made, are shown. $\Delta \rho_{A}$ is the value of $\rho_{A}$ calculated from observations on ambient parameters minus the gravimetric determination of $\rho_{A}$ based upon the above formula.

\begin{tabular}{c|c|c|c|c|c|c}
\hline \hline Test & $\begin{array}{c}\text { Number } \\
\text { of ob- } \\
\text { serva- } \\
\text { tions }\end{array}$ & $\begin{array}{c}\text { Range in } \\
\text { relative } \\
\text { humidity }\end{array}$ & $\begin{array}{c}\text { Range } \\
\text { in baro. }\end{array}$ & $\begin{array}{c}\text { Range } \\
\text { in temp. }\end{array}$ & $\begin{array}{c}\text { Average } \\
\Delta \rho_{A}\end{array}$ & $\begin{array}{c}\text { Std. dev. } \\
\text { in } \Delta \rho_{A}\end{array}$ \\
\hline & & $\%$ & $m m \mathrm{Hg}$ & ${ }^{\circ} \mathrm{C}$ & $\mu g / \mathrm{cm}^{3}$ & $\mu \mathrm{g} / \mathrm{cm}^{3}$ \\
First & 29 & 17.3 & 742.3 & 24.0 & -0.14 & 0.49 \\
& & 40.9 & 756.3 & 27.0 & & \\
Second & 42 & 7.3 & 746.0 & 21.5 & +0.35 & 0.33 \\
\hline
\end{tabular}

The above tests cannot be considered a "calibration" of our system because of possible errors in our values $\Delta M$ and $\Delta V$, and some other features which assume importance when "absolute accuracy" is a requirement. The tests demonstrate that placement of the sensors in the balance case is adequate and that the sensors have adequate sensitivity, stability, and speed of response.

\section{Apparatus}

The two most important pieces of apparatus necessary in hydrostatic weighing are the hydrostatic balance and the hydrostatic suspension. This chapter contains a section on each of these two members. In order to obtain a hydrostatic balance of adequate precision, we found it necessary to select an ordinary laboratory balance and modify it to this use. The criteria upon which selection of a balance should be made will be stated, and additionally we have suggested how an "ideal" hydrostatic balance might work. The hydrostatic balance can weigh the immersed load only to an accuracy which is limited by the ability of the hydrostatic suspension to deliver this load (without error) from the immersed object, through the air-liquid interface, to the balance. An adequate solution to this problem is described in this section.

\subsection{The Balance}

Precision. The precision of a balance is defined as the standard deviation of a single observation from the mean of many observations made by it on the difference between two loads. In a skillfully conducted sir weighing experiment of a given format of observations, the precision to which the difference between two loads is determined is almost entirely a function of the precision of the balance. In the air weighing cycle of the experiments reported here, precision is about $3 \mu \mathrm{g}$. In the water cycle, which includes almost all sources of variability occurring in the air cycle (plus many more) precision is believed to be about $4.5 \mu \mathrm{g}$, although this is difficult to prove experimentally. From this, it may be inferred that the variability introduced into the experiment by the balance is a major fraction of all variability. Therefore, the first criterion in the selection of a balance should be that it have an excellent precision as defined above.

Convenience. The second criterion in the selection of a balance is convenience of operation. In hydrostatic weighing the experimenter is a very busy individual indeed. He must monitor two extra temperatures not involved in air weighing ( 1 to about 0.001 ${ }^{\circ} \mathrm{C}$ ), he must manipulate two extra arrestments, he must keep the water surface in the vicinity of the meniscus free of dust, he must observe the immersed structure to guard against rotation, and he must maintain the format of observations in accordance with a previously chosen time schedule.

Mechanical smoothness of operation is the third selection criterion, and it is equally as important as precision and operating convenience. Arrestment and release must be accomplished with no jolts or vibration and with near zero horizontal pan motion. Many otherwise fine balances must be rejected for hydrostatic work because of arrestment or weight changer design. While significant irregularities of pan motion will exert no effect on air weighing, they can completely destroy a water weighing measurement. 
The importance of these three criteria cannot be overstated. Almer, Bowman, Jensen, Macurdy, Peiser, and Wasko [23] have pointed out how these criteria are best satisfied by a two-knife balance. We selected, as did Henins, a damped, single-pan, direct-reading balance almost entirely upon the basis of these three features. The balance we selected is designed to operate at a constant load of $20 \mathrm{~g}$. This amount of dialoperated weights is built into the suspension and may be removed in increments of $0.01 \mathrm{~g}$. When a load is placed on the pan, the built-in weights are removed from the suspension in a quantity sufficient to restore the balance to its design-load operating level. When the load removed from the suspension is within $0.01 \mathrm{~g}$ of the pan load, the beam swings to an angular position which indicates the residual unbalance.

Sensitivity is defined as the ratio between the angluar deflection of the beam and the magnitude of a load change on the pan. ${ }^{2}$ High sensitivity is desirable for clear and unambiguous observation on the scale of the difference between pan load and the load dialed-off from the suspension. In the Introduction we defined the apparent weight of an unknown object, $\bar{W}_{X}$, as the gravitational force exerted on it minus the buoyant force exerted on it by the surrounding medium. Thus:

$$
\bar{W}_{X}=\left(M_{X}-\rho_{A} V_{X}\right) g
$$

where $M_{X}$ and $V_{X}$ are the mass and volume of the object, $\rho_{A}$ the density of the medium and $g$ the local acceleration of gravity. Also:

$$
\bar{W}_{X}=M_{X}\left(1-\frac{\rho_{A}}{\rho_{X}}\right) g
$$

where $\rho_{X}$ is the density of the object. In a vacuum $\rho_{A}=0$, so

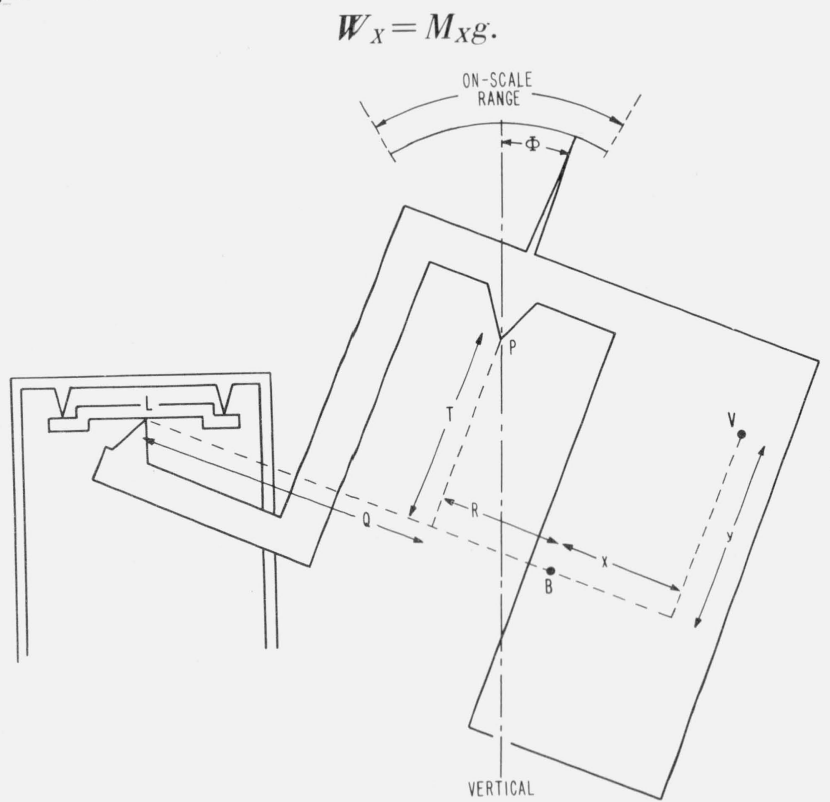

FigURE 2

${ }^{2}$ This ratio is best defined when the load change tends to zero.
Referring to figure 2, point $\mathrm{B}$ is the center of mass of the beam alone, at which point its apparent weight, $\overline{W_{B}}$, acts. Point L, a distance of $Q+R$ from $\mathrm{B}$. is the point on the load knife at which the compensating pivots concentrate $\bar{W}_{H}$, the apparent weight of the hanging load, which has two components:

(a) $\bar{W}_{s}$, the apparent weight of the suspension itself which includes whatever dial operated weights remain in place, and

(b) $\bar{W}_{L}$, the apparent weight of the load placed upon the pan. If the center knife is placed at point $\mathrm{P}$ with coordinates $(Q, T)$ from the origin, $\mathrm{L}$, along $\mathrm{LB}$, the system will rotate (assuming vacuum operation) and decay toward an angular position $\Phi$ described by the following equilibrium equation:

$\left(\bar{W}_{S}+\bar{W}_{L}\right)(Q+T \tan \phi)$

$$
=\bar{W}_{B}(R-T \tan \phi)
$$

Differentiation of this equation gives us immediately an expression for system sensitivity, $S$, thus:

$$
S=\frac{d \Phi}{d \bar{W}_{L}}=(-) \frac{\cos ^{2} \Phi(Q+T \tan \Phi)}{T\left(\bar{W}_{B}+\bar{W}_{S}+\bar{W}_{L}\right)} .
$$

This expression for sensitivity appears in numerous forms, depending upon the trigonometric description of the beam used in writing the equilibrium equation. By suitable algebraic manipulation, they can be shown to be equivalent. Most expressions for $S$ contain the ratio between the two moment arms or arm radii. These forms were originally written to apply to threeknife, symmetrical balances because, on such instruments, the effective value of the ratio can be determined gravimetrically (by transposition). Direct measurement of the individual arms sometimes leads to intolerable error in the ratio. When working values of the two arm lengths are required, the sum of the two arms (very nearly equal to the total distance between the two end knives) is obtained in one length measurement and this is solved simultaneously with the ratio. For high-sensitivity symmetrical twopan, three-knife balances of conventional design, individual values of the two arms can be obtained in this manner with acceptably small error.

This technique is impractical on a nonsyinetrical two-knife balance, so our system is described in terms of the ratio between $Q$ and $T$, figure 2 , thus:

$$
Z=\frac{Q}{T} \text {. }
$$

The effective value of $Z$ may be approximated on a two-knife balance. It is noted that the measurable distance between the two knives is $\sqrt{Q^{2}+T^{2}}$. When individual values of $Q$ and $T$ are demanded, they are available from a simultaneous solution of equations involving numerical values of $\frac{Q}{T}$ and $\sqrt{Q^{2}+T^{2}}$. It is 
noted that $Z=\cot$ PLB, however measurement of this angle is difficult.

If the total apparent weight of the system, $\overline{W_{T}}$, is the sum of the elements $\bar{W}_{B}+\bar{W}_{S}+\bar{W}_{L}$, then eq (7) becomes

$$
S=\frac{d \phi}{d \bar{W}_{L}}=(-) \frac{\cos ^{2} \phi}{\bar{W}_{T}}(Z+\tan \phi) .
$$

This shows that the system sensitivity is a function of the angular position of the beam with respect to gravity, and the dependence of $S$ upon $\phi$ is

$$
\frac{d S}{d \phi}=\frac{Z \sin (2 \phi)-\cos (2 \phi)-1}{\bar{W}_{T}}
$$

Maximum sensitivity and independence of $S$ from $\phi$ exist when $\frac{d S}{d \phi}=0$, under which circumstances $\cot \phi$ $=Z$ and

$$
Z=\bar{W}_{T}\left(S_{\max }\right) .
$$

Linearity and on-scale range are the fifth and sixth criteria in hydrostatic balance selection. It was pointed out in the introduction that the balance is used as a linear force indicator, and that the units of force indicated could be perfectly arbitrary, provided that they were consistent in the water-weighing and air-weighing cycles of the experiment. A large on-scale range is desirable so that a large difference can exist between the pan load and the removed dialoperated load. In addition to simplifying the operation (it is easier to read a difference than to dial it out) fewer dial operated weights become involved so calibration uncertainties are minimized. The requirements for large sensitivity and large on-scale range taken together demand that the balance swing over a very large range in $\Phi$. But eq (10) shows that $S$ is dependent upon $\Phi$, except at the single point defined in eq (11), so that linearity is sacrificed to achieve sensitivity and range.

There are techniques for providing uniform apparent sensitivity over the entire on-scale range (such as an observing system with compensating nonlinearity) and it does not matter to the experimeter what, if any, technique is used. He must, in any event, verify uniformity of sensitivity or evaluate nonuniformity by experiment. Figure 3 is a plot of the data taken on our balance, which shows the dependence of $S$ upon $\Phi$ shown in eq (10) as well as nonuniformity due to other causes. The on-scale range of our balance is $20 \mathrm{mg}$ for beam motion of $0.044 \mathrm{rad}$. The experiment consisted of plotting observed changes in $\Phi$ (expressed in milligrams) for the application of the same $5 \mathrm{mg}$ weight at various points in the on-scale range. Figure 3 shows our lack of linearity to be quite trivial, nevertheless, we constructed an empirical formula to eliminate this source of nonlinearity,

$$
s_{t}=s_{0}+\left(10-s_{0}\right)^{2}(0.00007) \text { milligram }
$$

where $s_{t}$ is taken to be the corrected screen reading

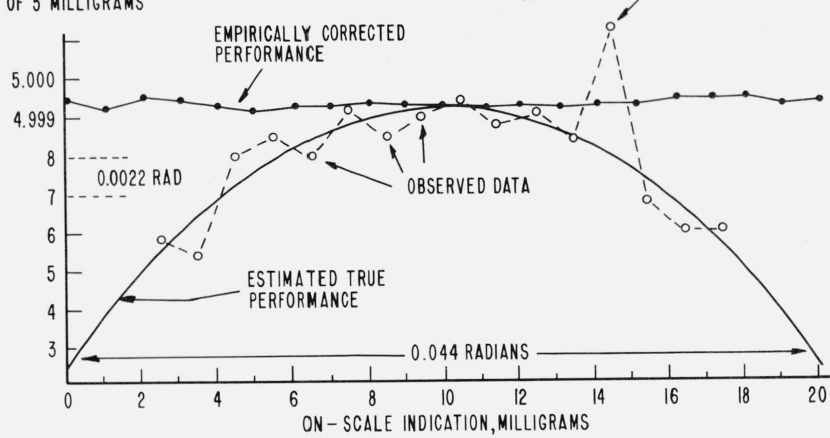

FigurE 3. This is the average of four sets of measurements on the sensitivity of our balance at various points over the on-scale range.

The open circles are observed data. For various reasons it is difficult to obtain reliable data at the end-points of the range. The solid line is our estimate of true performance, and the approximately horizontal line is the performance when observations are corrected by an empirical formula as explained in the text.

and $s_{0}$ the reading actually observed. It should be noted that if the balance is moved from one location to another, or if the leveling screws are changed, the characteristics of linearity will be altered, which would require a change in the empirical formula.

Another source of nonlinearity lies in the maladjustment of the built-in weights, for example, failure of a 10 -g weight to be precisely twice that of a 5 . This effect may be minimized by a simple calibration procedure in which a set of standard weights is "weighed" on the balance in various combinations utilizing all settings of each dial. This procedure, worked out by $\mathbf{M}$. Jones, NBS, and described in detail in the appendix, leads to a table of corrections which are applied to the dial settings on the balance. The corrected dial reading, $D_{t}$, is obtained by

$$
D_{t}=D_{0}+C
$$

where $C$ is the appropriate correction selected from the table, and $D_{0}$, the observed dial reading.

Load pan fall, $F$, is the vertical distance through which the suspended load moves as the balance swings through its on-scale range. For balances of conventional design

$$
F \cong Q \Phi_{T}
$$

where $\Phi_{T}$ is the total angle (in radians) through which the beam swings. The greater the value of load pan fall the longer must be the length of suspension wire which must be adequately coated. This is discussed later in detail, but it can be stated here that $F$ should be held to a minimum. Minimizing either $Q$ or $\Phi_{T}$ to reduce $F$, will, unfortunately, degrade either sensitivity or on-scale range. 
Buoyant Equilibrium. Equations (6) through (11) hold for operation in a vacuum. They also hold for operation in a medium of density $\rho_{A}$ if the balance is in buoyant equilibrium, by which we mean two requirements are met:

(1) The beam is homogeneous or effectively so. Effective homogeneity exists when the center of mass of the beam coincides with its center of volume.

(2) The net density of the suspension (the sum of all hanging masses divided by the sum of their volumes) equals the net density of the beam.

In the case of the suspension, effective homogeneity is not demanded, because both downward mass forces and upward buoyant forces are concentrated at point $\mathrm{L}$ in the diagram. Violation of the requirements for buoyant equilibrium results in changes in sensitivity and equilibrium values of $\phi$ with $\rho_{A}$ (zero drift) as explained below.

When requirement 1 is violated the center of volume of the beam, $V$, lies at coordinates $(x, y)$ from the center of mass at point $\mathrm{B}$, and the equilibrium equation is

$$
\begin{array}{r}
\left(\bar{W}_{L}+\bar{W}_{S}\right)(Q+T \tan \phi)=\left(M_{B} g\right)(R-T \tan \phi) \\
-g \rho_{A} V_{B}(R-T \tan \phi+x+y \tan \phi) .
\end{array}
$$

Differentiation of eq (14) to obtain an expression for balance sensitivity under these conditions gives:

$$
S=\frac{d \phi}{d \bar{W}_{L}}=(-) \frac{\cos ^{2} \phi(Z+\tan \phi)}{\bar{W}_{T}+\frac{\rho_{A} V_{B} g y}{T}} .
$$

This expression is seen to be identical to the previously developed sensitivity formula (eq (9)) except for the addition of the second term in the denominator which shows the various extraneous factors which exert an effect on sensitivity when the balance is in violation of requirement 1 for buoyant equilibrium. It is seen that $x$ has dropped out, a fact which tends to simplify adjustment.

Violation of requirement 2 . When the density of the hanging load, $\rho_{H}$, differs from that of the beam, $\rho_{B}$, air density, $\rho_{A}$, exerts an effect on $\phi$, thus:

$\frac{d \phi}{d \rho_{A}}=\frac{\cos ^{2} \phi\left[\frac{\bar{W}_{H}(Q+T \tan \phi)}{\rho_{H}-\rho_{A}}-\frac{\bar{W}_{B}(R-T \tan \phi)}{\rho_{B}-\rho_{A}}\right]}{T \bar{W}_{T}}$

When $\rho_{H}=\rho_{B}$ this function becomes zero, however, this is rarely the case, because the density of the load on the pan (which cannot be anticipated by the manufacturer) exerts an influence on the net suspension density, $\rho_{H}$. What the balance maker can do is adjust the density of his beam and of his suspension (with all built-in weights in place) to midrange between the maximum and minimum values of $\rho_{H}$ which is likely to be encountered in laboratory work. Our (loaded) suspension weighs about $50 \mathrm{~g}, 20$ of which are made up of built-in weights of density $7.75 \mathrm{~g} / \mathrm{cm}^{3}$. The densities of the suspension and beam are each about $4 \mathrm{~g} / \mathrm{cm}^{3}$. When the built-in weights are removed and replaced alternatively by a $20 \mathrm{~g}$ pan load of aluminum and then platinum, $\rho_{H}$ changes only from about $3 \mathrm{~g} / \mathrm{cm}^{2}$ to $5 \mathrm{~g} / \mathrm{cm}^{3}$.

The effects of buoyant inequilibrium are not always insignificant, and it is difficult to separate the effects of beam shortcomings and suspension shortcomings. What one can demand, however, is that the "observed zero" on the calibrated scale and sensitivity be independent of $\rho_{A}$. We observed our system on days of high values of $\rho_{A}$ and low. We could not detect a systematic relationship between $\Phi$ and $\rho_{A}$ over the limited range of $\rho_{A}$ available to us because of other sources of zero drift. Out of curiosity we placed our balance in a closed system and reduced $\rho_{A}$ from about $0.0012 \mathrm{~g} / \mathrm{cm}^{3}$ to near zero by evacuating it. The beam rotated to indicate an increase in $\bar{W}_{L}$ of about $1700 \mu \mathrm{g}$ which was taken to indicate adequate buoyant equilibrium. This test is unnecessary and is not recommended.

Damping. A direct-reading balance used in airweighing should be damped so that the beam swings quickly to its equilibrium position and thereafter remains motionless. Viscous (i.e., air) damping is used on most direct reading balances. A person not familiar with balances is usually shocked at the apparently gigantic size of the damper, considering the vanishingly small forces to be damped. The answer lies in the clearance which must be left between the damping vane and dash pot. The clearance must be large, because a particle of dust caught between these members could completely destroy the precision of the system. However the damping vane cannot be made indefinitely larger because zero drift (resulting from sail effect) would become intolerable. Damper requirements are minimized when the moment of inertia of the system is minimized, and also (for any given beam frequency) sensitivity is maximum. Moment of inertia is minimized when both the load arm and counter-weight arm are minimum. In our system, both arms are held to within a few millimeters of $5 \mathrm{~cm}$. The damping vane is about 1 in in diameter, and zero drift is rarely more than a few micrograms per minute. In hydrostatic operation, the damping associated with the immersed load is far greater than that obtained during an air weighing, so air damping is not an essential feature of a direct reading balance used only in hydrostatic work.

The ideal hydrostatic balance. From the foregoing comments it is obvious that the criteria upon which a direct-reading hydrostatic balance is judged differ significantly from criteria applied to air-weighing systems. Equation (7) shows that the principal masses in the system (the beam, the suspension, and the load) are equally important in the static situation; but in the distribution of total mass, the designer must operate under dynamic and engineering-type restrictions. For maximum sensitivity and beam frequency, minimization of moment of inertia is called for. He obviously wants the useful load to be a large fraction of 
the total, which demands a suspension sufficiently massive to support the load and a beam sufficiently massive to support the loaded suspension and maintain the integrity of triangle LPB. As total mass increases, sensitivity is lost. Increasing the $Z$ ratio to regain $S$ results eventually in loss of adequacy of rigidity. Decreasing total size to improve $S$ results in knife imperfections assuming importance. Attempts to decrease $F$ result in degradation of sensitivity and range.

It occurs to us that many of these restrictions would disappear in a system in which the beam is driven to a null position (probably $\cot \Phi=Z$ ) by a servo-controlled force. Almer and Bowman [4] have pointed out how such an arrangement will minimize arrestment and knife-edge problems. Various corrections as well as damping could be inherent in the feed-back loop so zero drift could be reduced.

This would give the designer great latitude in nonsymmetrical configuration. Torsion fiber balances may have on-scale ranges much larger than that of our balance. If the major load were supported by knife edges and the servo-controlled torque applied by means of a torsion fiber, there would be an enormous increase in sensitivity, linearity, and on-scale range. It would be quite practical to restrict mechanically the beam to a very small $\Phi_{T}$, so that $F$ would be very nearly zero, thereby minimizing many suspension wire problems. With greater on-scale range, an entire decade of built-in suspension weights could be eliminated (along with their calibration uncertainties). Elimination of the smallest decade $(0.01 \mathrm{~g}$ to $0.1 \mathrm{~g})$ would make it possible to use ring weights centered on the suspension for dial operation. This would minimize the horizontal suspension forces associated with offcenter dial-operated weights.

Many years ago, H. S. Peiser, NBS, proposed by private communication total immersion of a torsion fiber balance in the hydrostatic fluid as a means of eliminating meniscus uncertainties and minimizing the thermal drift in the balance. Certain practical problems prevented pursuit of this approach; however, the same basic idea could be applied to a force measuring system built around a load cell. If the cell itself were immersed in the hydrostatic fluid, there would be no air-water interface, and the entire meniscus uncertainty would be eliminated from the measurement. Semiconductor strain gauges of adequate sensitivity are currently on the market. Their principal drawback is temperature dependence. Immersion of the cell in the (very well thermostated) fluid would minimize uncertainties from this cause.

These comments are largely speculative but not entirely so. We experimented with an electronically controlled equal-arm balance. Although it was not a nullbalance type servo-control (it was an electronic spring type) we were delighted at the speed of response, stability, damping and small load pan fall. For various reasons, not pertinent here, the precision was inadequate. NBS staff members have experimented with large size load cells $(1000 \mathrm{~kg})$ in certain high precision weighing situations, and found that when properly used, they can be made to rival the performance of high quality balances.

Balance modifications. Whereas Henins used his balance in the "as delivered" condition, we made a few minor modifications to ours to adapt it to the hydrostatic situation.

(a) Performance is degraded when the balance door is opened. In the water-cycle of our experiment the door must be opened twice during each observationfirst to place a sensitivity weight on the pan, and second to remove it. In the air cycle two of the four door openings in each observation are for manipulation of this weight. We drilled a small hole in the door of the balance to permit placing this weight on the pan and removing it by a rotating rod-thereby eliminating four of the six necessary door-openings.

(b) When the relative humidity in the laboratory was quite low (in the winter) we had problems associated with surface evaporation in the vicinity of the meniscus. To minimize this, a small dish of water was placed below the balance and near the hole in the bench through which the suspension to the hydrostatic fluid led. This required raising the legs of the balance by about 2 in to accommodate the dish. At the same time the open space between the bottom of the balance and the bench top was sealed by aluminum foil held in place by masking tape to protect the exposed hydrostatic suspension from drafts.

(c) Measurements made on balances of this type are based upon the assumption that the apparent weight removed from the suspension by dialing is equal to the apparent weight of the pan load (except for the indicated difference on the scale). Apparent weight by definition is a function of air density, so we drilled holes in the top of the balance to receive temperature and humidity sensors for the upper balance compartment where the dial operated weights were stored. Sensors for the pan compartment were already in place. We found that the air density in the upper compartment was usually within a microgram per cubic centimeter of that in the pan compartment. Inasmuch as the built-in weights displaced about $2 \mathrm{~cm}^{3}$, the systematic error involved was only marginally significant. Great effort was expended to assure that the upper and lower temperature and humidity sensors did not carry a residual bias.

\subsection{Hydrostatic Suspension}

This assembly delivers to the balance alternately the "pan-loaded" information and the "pan-empty" information, the difference being the apparent weight in water of the unknown object, $g\left(M_{x}-\rho_{w} V_{x}\right)$, which is used in our simultaneous solution for $\rho_{x}$. It hangs below the balance pan, passing through a hole in the floor of the balance case, penetrating the water surface, and extends to a depth of a few inches below the water surface. The portion of the assembly which is actually supported by the balance pan must be considered as part of the load capacity of the balance, so the heavier the load portion of the suspension assem- 
bly, the smaller the maximum allowable load which we may test. This assembly has several components which will be discussed separately, commencing at the bottom.

The sample unloading device, as the name implies, is the mechanism which removes the unknown object from the immersed pan when it is desired that the balance report on the loading under the "pan-empty" condition. This assembly is made of stainless steel in hopes that no water contamination will result from its surfaces. Figure 4 shows it to be a pair of linear

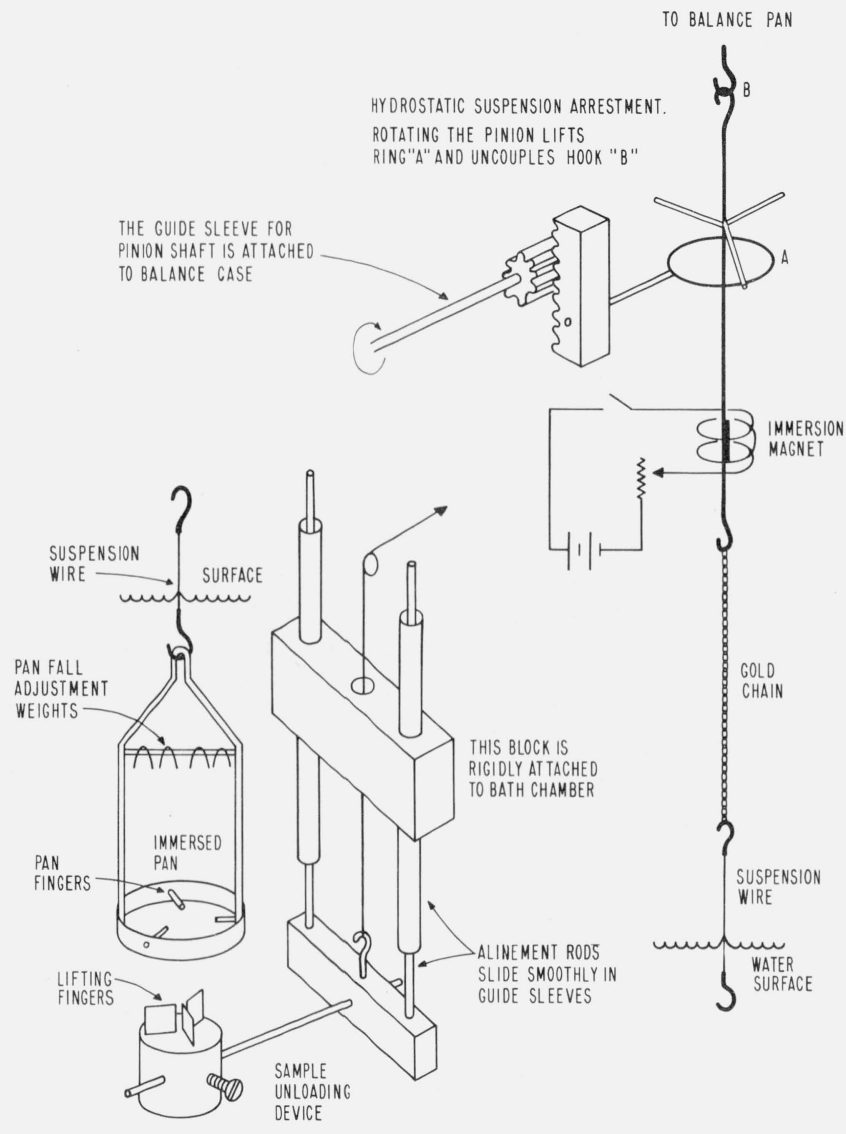

Figure 4

motion guides alined parallel so that the fingers which lift the unknown object from the pan will move approximately vertically. It is rigidly attached to the hydrostatic bath which has all degrees of freedom of position adjustment. The fingers which carry the unknown load when it is off of the pan form a plane, and this plane must be adjusted (by chamber adjustment) so that it is parallel to the plane formed by the pan fingers which carry the unknown load when the load is on the pan. In this way the unknown load is lifted smoothly off of the pan without tilting, and redeposited on the pan as required by the experiment.

The immersed pan hangs at the bottom of the suspension. It is made of brass to which are soft soldered the copper wire fingers which carry the unknown load during the "pan-loaded" cycle. The entire assembly is chrome-plated to avoid the contamination of the water by any oxides which may form on its surfaces. The plane formed by the pan fingers is adjusted to the horizontal by tiny wire weights and bending of the fingers, when the pan is empty and suspended by the top hook. This adjustment is important, and when once made need not be repeated. The intent of this adjustment is to achieve a horizontal pan finger plane, and when the lifting finger plane is made parallel to it, both planes are horizontal. Under these conditions, raising and lowering the lifting finger plane, by manipulating the sample unloading device, transfers the load of the unknown object alternately from the pan to the sample unloading device. If this load exchange is performed several times before the start of the experiment, the unknown load will move horizontally until its effective center of mass lies vertically below the suspension, at which time horizontal motion ceases. Thereafter the load may be lifted from the pan completely free from shock due to malalinement or tilting of the sample, both of which cause horizontal motion of the suspension which results in serious variability in data.

The suspension wire. Variability in the (downward) surface tension force associated with the meniscus surrounding the point of penetration of the water surface by the suspension wire has always been a major limitation in high reproducibility hydrostatic work. The problems become much greater as the size of the sample is reduced as the following argument shows:

(a) The actual magnitude of the force for a water meniscus is about 72 dyn per centimeter length of the meniscus, which is approximately equal to the circumference of the wire; therefore the wire is properly made no larger than is actually required to support the tensile load.

(b) If the load is reduced by a factor of four, the minimum acceptable cross-section area is also reduced by this factor, but under these circumstances the circumference is reduced by only a factor of two. Thus, if the percentage reproducibility of the surface tension force remains constant, the actual variability (in terms of total load) is doubled.

The above remarks are probably too optimistic, because the effective length of the meniscus is greater than the circumference of the wire, therefore the meniscus length actually decreases at a rate slower than the circumference. Additionally, since the total surface tension force is not distributed evenly around the meniscus (microscopic examination shows it may climb higher in some spot than others), an averaging process operates more efficiently on a long meniscus than on a short one.

The magnitude of this force for a wire 0.001 in in diameter is about 0.6 dyn. Inasmuch as we are attempting to measure total force to 0.003 or 0.004 dyn, severe requirements are placed upon meniscus stability, especially when it is considered that convective and turbulent water motion can account for a large part of this allowed variability. For years, experimenters have depended upon rough coatings 
of gold, platinum, and other materials electrically deposited on the wire surface, so that water would flow up the sides of the wire by capillary attraction, thereby forming a zero (and hence reproducible) contact angle. These devices work, but not reliably.

We experimented with numerous suspension wires of various materials with coatings formed by several electrochemical techniques. About 25 percent of these wires were found to contain a section which provided meniscus stability to about 0.01 dyn. The usable sections of these wires were photographed, and it was noted that in each case the coating had a fairly fine granular structure uniformly covering the wire.

Figures $5 \mathrm{~A}$ and $5 \mathrm{~B}$ are photomicrographs of different sections of the same piece of wire treated by classical techniques to achieve the desired granular rough surface illustrated. When the meniscus was
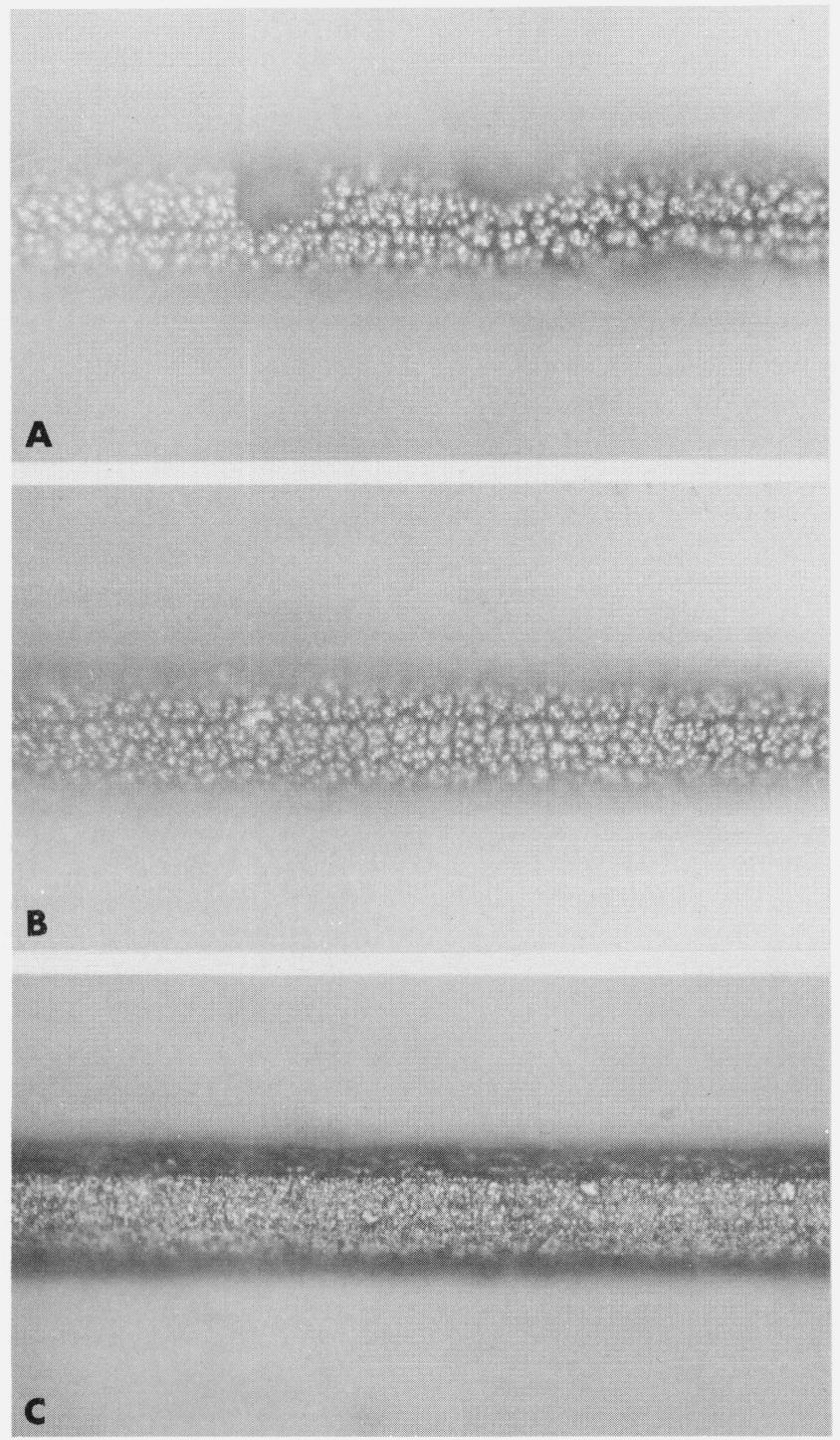

Figure 5 formed around the section shown in figure 5A, the hydrostatic performance was inadequate. After lowering the level of the water in the weighing chamber several times to permit the meniscus to form around different sections of the wire, a spot was finally located where performance was satisfactory. This section is shown in figure $5 \mathrm{~B}$. The difference between good and bad performance is ascribed to the presence of spots on the wire where the rough coating either did not form or where, after formation, it flaked off. Formation is inhibited when one of the tiny gas bubbles, which form on the wire surface during electrodeposition, clings to the wire rather than breaking away and rising to the surface of the electroplating bath. To prevent these bubbles from remaining on the wire surface it is customary to agitate mechanically the wire during the plating process; however the agitation itself will frequently cause the fragile coating already formed to flake away, leaving a bare (and hence unsatisfactory) surface. Unsatisfactory plating also results from inadequate control of electric current density at the plated surface and from improper solution strength or process temperature. After plating, the wire must be thoroughly cleaned to eliminate traces of the electroplating solution, otherwise contamination of the hydrostatic weighing water results. Henins followed the plating and cleaning processes with a careful optical examination of the wire surface at $200 \mathrm{X}$. The fabrication of a wire by classical techniques to these performance tolerances is a task requiring much patience, skill and luck.

We experimented with several alternative methods of wire fabrication and found that the most simple technique was the most reliable. A coating of chromium oxide was formed on the surface of nichrome wire by electrically heating it to a medium red heat in a 1 to 10 $\mu$ vacuum for about an hour.

While hot, the oxide coating is smooth, but when the electricity is turned off the parent metal cools and contracts, resulting in the brittle oxide coating breaking up into the desired surface structure. Such a wire is shown in figure 5C. It is evident that the surface is much more uniformly granular than in figures $5 \mathrm{~A}$ or $5 \mathrm{~B}$, and that the grain size is significantly smaller.

During the past year several dozen wires have been made by this technique and every wire performed adequately. In about one-half of the tests the first section of wire tried out gave satisfactory performance. In the other half it was necessary to drop the water level a few millimeters (so that the meniscus formed on a different section of the wire) to find a section of adequate performance. We have rarely been required to try out a third section. This is in sharp contrast to performance of electrically coated wires which frequently (after trying out many sections) turn out to have $n o$ adequate section whatsoever.

This process has an important by-product. During the heating cycle, all traces of dirt and grease are burned away, and it is possible to place the wire in service immediately upon completion of fabrication 
without an intermediate cleaning process or optical examination.

Our procedure for making hydrostatic suspension wires is as follows:

(a) Two hooks are bent from \#18 copper wire, figure 6 , and a piece of $0.001^{\prime \prime}$ nichrome wire tied between them. After tying, the nichrome length should be $6 \mathrm{~cm}$.

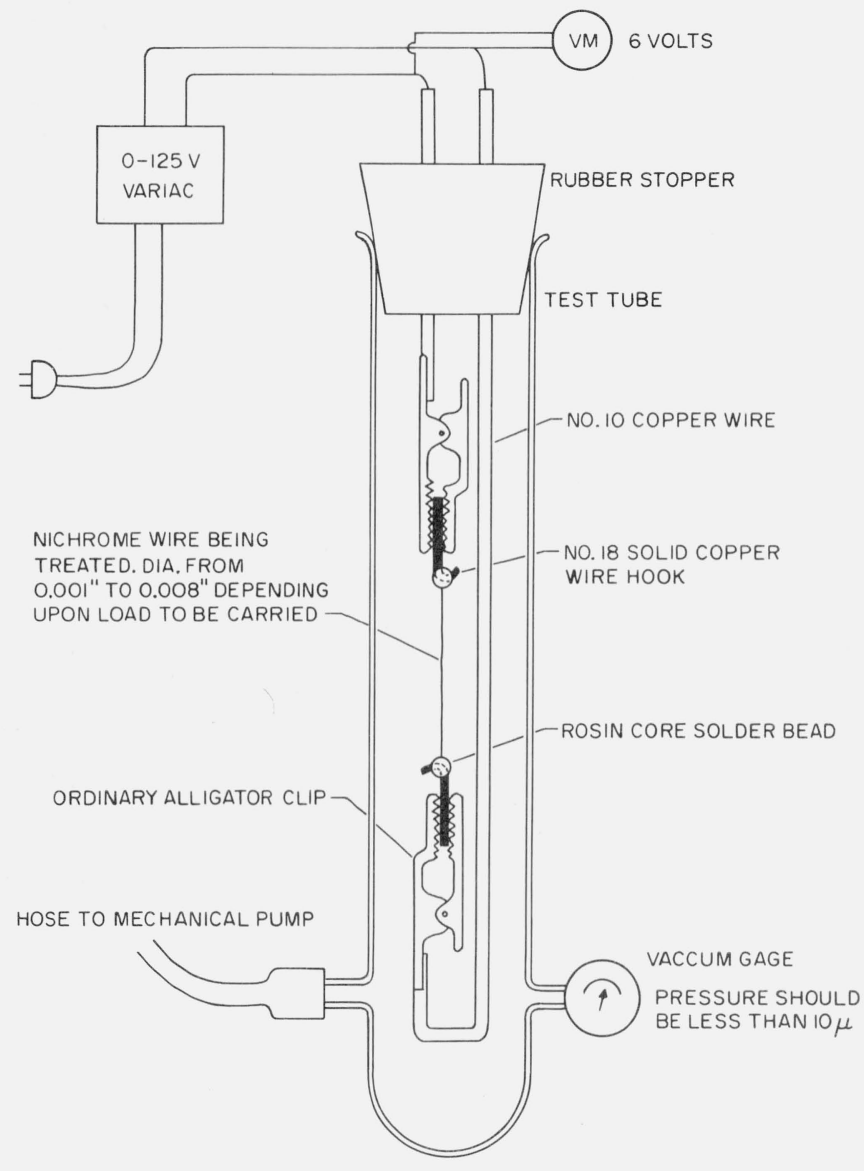

Figure 6

(b) Using rosin core solder, a bead is made at each hook. Although this solder does not wet the nichrome, it holds it firmly in place mechanically. The unit is soaked in benzene to remove the rosin.

(c) It is then placed in a holder built into a rubber stopper which may be inserted into a vacuum system and electrical leads are attached (see fig. 6).

(d) The vacuum pump is turned on and when the pressure is down to 1 to $10 \mu$ the electricity (set to $6 \mathrm{~V} \mathrm{ac})$ is turned on.

(e) After $1 \mathrm{hr}$ the pump and current are turned off and the wire is put into service.

We have recently found that if the wire is initially wet with a stream of steam just before placing it in water the performance is improved. Although this is a tempting path of further investigation, other errors of greater magnitude demand attention.

The immersion magnet. This is a very useful con- tribution to the art from Henins. It is a tiny wire magnet weighing perhaps $0.2 \mathrm{~g}$ which is cemented to the suspension link between the upper hook of the suspension wire and the hydrostatic suspension arrestment. Surrounding the suspension in the vicinity of this magnetic wire is a 30-turn electric coil which when energized causes the suspension to dip a few millimeters. Henins used this device as a test of proper functioning of the suspension wire. In such service it is excellent however, as will be explained later, this is only one-half of the test. That is, a wire which fails this test will not function at the position tested; however wires which do pass this test may fail on even more critical tests later. The tests are quite simple (10 min maximum to find a satisfactory spot) if the proposed method of wire treatment is used. Before attaching a magnet to the suspension system of a balance, one should verify that ambient field changes will not cause significant balance drift. In one of our experiments, nonreproducibility was traced to field changes caused by work in a nearby laboratory.

The hydrostatic suspension arrestment is the device which uncouples the hydrostatic suspension from the balance for air-weighing operation. Its design is not critical, the only requirements being that:

(a) It operate without mechanically shocking the system, and

(b) arresting and releasing the hydrostatic suspension results in reproducible coupling of the suspension to the balance pan. Our design is shown in figure 4 .

\section{Sample Handling and Degassing}

It is generally recognized that air adhering to the surfaces of the test sample is a major source of nonreproducibility in independent density measurements. If this surface-trapped air were a random phenomenon, the only effect would be to degrade the standard deviation of a single observation, and exert little effect on the mean value of several observations. However, surface air causes a systematic effect in that it always acts to make the measured value of $\bar{W}_{w}$ (and hence the calculated density) appear lower.

A bubble of air large enough to displace $1 \mu \mathrm{g}$ of water can be removed after detection by a suitable optical arrangement. On the other hand, the same amount of air dispersed among a large number of very tiny bubbles would go completely undetected except for its derogatory effect on precision.

The method initially used for comparison of degassing techniques was to measure the density of samples after being degassed by various methods. The difficulty in this method was that as degassing methods improved, the indicated density changes became smaller and finally, when they became of the order of the experimental variability, they were 
impossible to detect without an impractically large number of observations.

We were fortunate to have a Cartesian diver whose standard deviation was significantly lower than that of classical hydrostatic systems, which we could use for comparison of degassing techniques. Actually, the problem was first attacked because surface gas was seriously degrading Cartesian diver data.

Our Cartesian diver system [3] provides values of $\bar{W}_{w}$ to a much higher precision than the system described here but with inordinately greater effort. Additionally, values of $\bar{W}_{W}$ are not degraded by meniscus uncertainties inasmuch as it is a completely immersed system. Since it operates at $3.98{ }^{\circ} \mathrm{C}$ slight errors in thermostating do not cause significant variability in $\rho_{W}$. Variability in values of density calculated from diver data is caused almost entirely by variability in surface-bound gas on the sample.

In these experiments we used two thin disks of single crystal silicon. This geometric form is ordinarily avoided in hydrostatic work because of the relatively large surface area upon which gas may adhere, but in this experiment we particularly wanted a large area to magnify the effect.

Three series of experiments were performed: The first, in which the crystals were cleaned by the technique used by Henins, that is, by immersion of the crystal in water below a vacuum; the second by ordinary outgassing for $24 \mathrm{hr}$ under a $1 \mu$ vacuum and a temperature estimated to be about 75 to $100{ }^{\circ} \mathrm{C}$ from an infrared heat lamp; the third series of experiments utilized a boiling technique to be described.

Between each measurement in a given series, both crystals were removed from the hydrostatic system, exposed to air, dried, and recleaned and degassed. Ordinarily they would have been air weighed between each measurement, but we wanted to eliminate any variability in the calculated value of $\rho_{X}$ from variability due to this source, so they were air weighed only once for each series, immediately prior to the first hydrostatic experiment in the series, The data is shown below.

\begin{tabular}{l|r|r|r|r|r|r}
\hline & \multicolumn{2}{|c|}{ Henins method } & \multicolumn{2}{c|}{ Heat and vacuum } & \multicolumn{2}{c}{ Boiling } \\
\cline { 2 - 7 } & Sample 1 & Sample 2 & Sample 1 & Sample 2 & Sample 1 & Sample 2 \\
\cline { 2 - 7 } Test 1 & 2.3289957 & 2.3289972 & 2.3289937 & 2.3289991 & 2.3289950 & 2.3290012 \\
Test 2 & 2.3289970 & 2.3289986 & 2.3289975 & 2.3289997 & 2.3289958 & 2.3290018 \\
Test 3 & 2.3289930 & 2.3289972 & 2.3289964 & 2.3290013 & 2.3289944 & 2.3290017 \\
Test 4 & 2.3289965 & 2.3289980 & 2.3289959 & 2.3290013 & 2.3289950 & 2.3290015 \\
Test 5 & 2.3289946 & 2.3289964 & & & & \\
\hline Std. dev.......16 & 08 & 16 & 10 & 06 & 03 \\
\hline
\end{tabular}

The above data show that the boiling technique results in a standard deviation in density significantly lower than either of the other two techniques which was most gratifying because the boiling procedure is far simpler than either of the other two methods. It is also noted that the standard deviation of measured values of density of sample \#1 is always about twice as great as that for sample \#2, regardless of the degassing technique used. We frequently observe this phenomenon, and whenever it has occurred previously, a careful microscopic examination has always disclosed a crack or crevice in the sample. A most methodical examination failed to disclose such a shortcoming on the surface of sample \# 1 in these experiments and we can offer no explanation for this observed effect.

The boiling technique, which is shown by the above data to provide the best degassing of the three methods being compared, is performed as follows:

(1) The sample is immersed in a small vessel containing room-temperature (but previously boiled) water.

(2) Using a hot plate or very low flame, it is brought slowly to a boil and allowed to boil for several minutes. At the end of this boiling interval, the boiling should be quite vigorous.

(3) The water is cooled to a fairly low temperature with the vessel covered. The lower the temperature, the better as long as there is no freezing.

We have the impression that there are two mechanisms involved in this boiling process. First is that steam will form on particles of surface-trapped air as though the air were acting as a seed. When boiling is "just" commencing, many tiny trains of steam bubbles can be seen originating from stationary discreet points on the sample surface. These points do not wander around on the surface; however, without warning, a train of bubbles will stop forming. We take this to indicate that the train of steam buhbles has either removed the air gradually or that the mechanical agitation associated with the boiling has broken it loose from the sample surface and permitted it to rise to the water surface. The second mechanism believed operating is that in the cooling of the water it becomes a better solvent for any residual surfacetrapped air.

We are unable to explain why the boiling associated with the vacuum in the other two processes fails to act as efficiently as the boiling associated with heating. It is possible that the cleaning procedures used to clean samples prior to density measurements leave a grease coating on the sample which holds air between the coating and the sample, and that heating melts the grease and permits the air to escape.

This degassing technique has been used in connection with density measurements on rutile, fused silica, nichrome, gold, platinum, and titanium in addition to single crystal silicon. No systematic effect on the efficiency of this method as a function of material was noted and we believe, without proof, that it will perform adequately on many materials for which density measurements by water weighing are appropriate.

\section{Weighing Procedure}

In this section we are concerned with obtaining values of $0_{A}$ and $0_{W}$, the balance observations when 
the object of interest is immersed in air and water respectively, from which the values of $\bar{W}_{A}$ and $\bar{W}_{W}$ are calculated. For various reasons to be explained, the air weighing should be performed first.

\subsection{Air Weighing Cycle}

After cleaning, the object of interest should be kept in the balance case several hours to come in to thermal equilibrium with the surrounding air. The air weighing cycle of the experiment consists of observing the balance under five conditions.

(a) The balance pan empty.

(b) The pan loaded by the object of interest.

(c) The pan loaded by the object of interest and also by the remotely controlled sensitivity weight discussed in section 4 .

(d) The object of interest removed from the pan, but with the sensitivity weight remaining in place.

(e) The sensitivity weight removed, that is, a repeat of the first condition.

Under each of the above five conditions we obtain an observation of the dial indication, $D_{0}$, and an accompanying screen observation, $s_{0}$. Each value of $D_{0}$ is corrected into a value of $D_{t}$ by eq 13 . If the screen is assumed to be adequately linear the five values of $s_{0}$ may be used as recorded, in which case they are named $s_{t}$ rather than $s_{0}$. If tests have shown the screen to be nonlinear, then an appropriate correction must be applied to each $s_{0}$ to convert it into $s_{t}$, in a manner comparable to eq (12) (which applies only to our balance).

The first, fourth, and fifth values vi $D_{t}$ are equal as are the second and third, thus:

$$
\begin{aligned}
& D_{t 1}=D_{t 4}=D_{t 5} \\
& D_{t 2}=D_{t 3} .
\end{aligned}
$$

This reduces to two $\left(D_{t 1}\right.$ and $\left.D_{t 2}\right)$ the number of dial readings which must be operated upon. These two values of $D_{t}$ and the five values of $s_{t}$ are combined in the following formula to provide a value of $0_{A}$, the balance observation in the air cycle:

$$
0_{A}=D_{t 2}-D_{t 1}+\frac{G\left(S_{t 2}-s_{t 1}+s_{t 3}-s_{t 4}\right)}{s_{t 3}-s_{t 2}+s_{t 4}-s_{t 5}}
$$

where $G$ is the mass of the sensitivity weight (in our experiment $G=0.0049992 \mathrm{~g})$. Use of this five-observation format, reduced as shown in eq (17), tends to minimize the effects of balance drift on the value of $0_{A}$. In our work we always carried out this entire procedure four times, thereby obtaining four values of the balance observation and averaged them to obtain a single value of $0_{A}$. In conjunction with these air weighings, temperature, barometric pressure and relative humidity were observed and recorded for later calculation of air density.

\subsection{Water Weighing Cycle}

When the various parts of the immersed structure are thoroughly cleaned, they should be assembled as described in section 4 . Following this, the water weighing chamber should be filled with distilled water. In the filling process air bubbles will be trapped on most of the immersed surfaces. After filling, we have found it convenient to boil the entire system for a few minutes with immersion heaters to remove this air. After boiling, the system requires several hours (usually overnight) to attain thermal stability. The water weighing procedure is divided into four distinct groups of tasks, the first three of which may be performed during the latter part of the stabilizing period: (a) degassing the sample, (b) adjusting the system, (c) testing the system, (d) making the observations.

(a) Degassing the sample should be performed as described in the immediately preceding section. Then the sample should be transferred, while completely immersed in a dipper, into the hydrostatic bath. During this procedure, the balance should be arrested and the hydrostatic suspension arrestment should be in the "release" position. The immersed pan unloading device should be in the "unload" position and the sample should be placed on the lifting fingers of this devicenot on the immersed pan fingers. During this loading process, a small amount of off-temperature water will be added to the system (from the dipper) which will change the bath temperature. Inasmuch as the bath has presumably not yet come into equilibrium, this additional upset causes no extra waiting; however, this loading procedure should be performed early in the experiment.

(b) Adjusting the system should be performed late in the waiting period because, although there are several tasks involved, individually they require only a few minutes each. There should be a smooth transition between these preliminary tasks and the primary task of making the observations. These comments hold only for a freshly filled system. We find that we are able to operate a system for four or five days before it becomes so corrupted by dust and dirt that recleaning is necessary. For a previously filled system, steps (b) and (c) may be performed as soon as the P.R.T. (platinum resistance thermometer) indicates that the thermal shock associated with loading has dissipated. The objective of the tasks in group (b) is to achieve a system adjustment such that the sample may be loaded and unloaded from the immersed pan and the dialoperated weights in the balance may be changed without mechanically shocking the system. These tasks are performed as follows:

(1) The sample should be loaded and unloaded from the immersed pan several times until its center of gravity is located directly below the vertical axis of the hydrostatic suspension. This is easily accomplished by external manipulation of the immersed pan unloading device as discussed in section 4. This process is performed with the balance arrested and the hydrostatic suspension arresting device in the "release" position.

(2) The operator should next release the balance in accordance with the manufacturer's instructions and determine by experiment the dial positions which 
place the system in on-scale equilibrium for both the immersed pan-empty and the immersed pan-loaded conditions. Even the best direct-reading balances have residual malalinement of the built-in dial-operated weights, and the operator will find that certain combinations (dial positions) will result in violent kicking of the suspension when released. We have gained some experience in overcoming this difficulty and possible remedies are discussed in the section on Operating Hints. Manufacturers give scant attention to this problem because it is bothersome only to experimenters doing water weighing. The effect of this weight misalinement appears to be negligible in ordinary air weighing.

(3) The final step in group (b) is to clean the water surface in the vicinity of the meniscus. This is accomplished by opening a port in a glass tube surrounding the suspension (to protect it from air currents) and vacuum cleaning the surface with a drawn-out glass tube. This is critical inasmuch as a particle of dust at the meniscus will destroy its reproducibility. We have found that back-lighting this surface makes specks of dust floating on the surface stand out clearly in the specular light.

This concludes the system adjustment. When properly performed, it should be possible to load the immersed pan and set the dials, so that when the balance is released it moves smoothly to an indication on the screen. Then after arrestment and immersed pan unloading, the dials should be set to predetermined positions and upon balance release there should be no mechanical shock to the hydrostatic suspension and the balance should move smoothly to a screen reading within about a few milligrams of the previous reading.

(c) Testing the system is the last of the preliminary steps prior to observations. As previously mentioned, there should be a smooth transition from this step to the final step of making the observations. In other words, as soon as these tests indicate adequate performance, testing (as such) is terminated and observations begin. The test procedure is very similar to the observing procedure, but the operator must not use the "last few" test runs as observing runs since this is comparable to data selection in which case the statistical analysis of the results would be put in jeopardy. In the system test procedure, we are basically testing the operation of our suspension wire. We have stated that 100 percent of the wires made by the procedure described have contained a section of adequate surface roughness and uniformity to provide high quality water weighing data. On the other hand, we have found that in about 50 percent of the cases the first section of the wire we attempt to use is inadequate and we must lower the water level a bit to expose a different section. We have occasionally had to try out a third section, but, to date, we have not had to make a fourth try. The system test to be described is basically a quick method of determining whether the section of wire being tried out is adequate for the task. It is performed in two steps, the first is a rough test only, using the suspension magnet (fig. 4) to deflect the balance, but in the second step the built-in sensitivity weight is actually used as in the final measurements.

When we are ready to start the system test procedure, we release the balance with no load on the immersed pan and we adjust the magnet current to dip the hydrostatic suspension deeply enough to cause the balance screen reading to increase by about 10 to $12 \mathrm{mg}$. The magnet is energized a few times to insure that the section of wire immediately above the section to be tested is thoroughly wetted by the hydrostatic water. The test is then performed as follows:

(1) The balance is released and the screen reading is observed and recorded.

(2) Without arresting the balance, the magnet is energized until the "deep" position is attained, after which it is de-energized and the balance permitted to return to its normal position. The screen is again read and recorded.

(3) Steps 1 and 2 are repeated several times, arresting and releasing the balance each time after making the observation called for in step 2. If the differences between the observations recorded in steps 1 and 2 are constant to within a few micrograms, we may proceed to step 4. If they are not constant, we may assume that the active section of the wire surface is inadequate and the water level is lowered a few millimeters to expose a new surface, and the above tes ${ }^{+}$procedure is repeated. When an adequate section of wire is located, we proceed with step 4 .

(4) In step 4 the balance is observed under each of 5 conditions, and in each condition a value of $D_{0}$ and an accompanying value of $s_{0}$ is taken.

(a) The balance is released and observed in the "immersed pan empty" condition, after which it is arrested.

(b) Using the immersed pan unloading device described in section 4 , the sample is placed on the immersed pan. The balance is then released and observed, after which it is arrested.

(c) The sensitivity weight is then loaded by the remote linkage. The balance is then released and observed, after which it is arrested.

(d) Using the immersed pan unloading device, the sample is removed from the immersed pan. The balance is then released and observed, after which it is arrested.

(e) The remote control sensitivity weight is removed from the pan. The balance is then released and observed, after which it is arrested. This is a repeat of the first condition.

(5) Values of $D_{0}$ and $s_{0}$ recorded during the water weighing cycle are corrected into values of $D_{t}$ and $s_{t}$ in the same manner as described above in "Air Weighing Procedure." Again $D_{t 1}=D_{t 4}=D_{t 5}$ and $D_{t 2}=D_{t 3}$, so we confine operations to only two $D$ 's and five $s$ 's. These are combined into a value of $0_{W}$, the balance observation in the water cycle, as follows:

$$
0_{W}=D_{t 2}-D_{t 1}+\frac{(G)\left(s_{t 2}+s_{t 3}-s_{t 1}-s_{t 4}\right)}{\left(s_{t 3}+s_{t 4}-s_{t 2}-s_{t 5}\right)} .
$$


The system is judged to be performing adequately when successive values of $0_{W}$ repeat to within about $5 \mu \mathrm{g}$.

(d) The observations are made in the exact same manner as described immediately above. We repeat the process four times to obtain four values of $0_{W}$ which are averaged to a single value.

(e) While making the observations leading to $0_{W}$, the parameters necessary for the calculation of air and water densities are recorded.

As stated in the introduction we are interested in obtaining the apparent weight of the sample in media of two different, but known, densities. The apparent weights of the object in air and water, $\bar{W}_{A}$ and $\bar{W}_{W}$, respectively, are given by

$$
\begin{aligned}
& \bar{W}_{A}=0_{A}\left(1-\rho_{A U} / \rho_{s}\right) \\
& \bar{W}_{W}=0_{W}\left(1-\rho_{W U} / \rho_{s}\right)
\end{aligned}
$$

where $\rho_{s}$ is the density of the built-in weights $(7.75$ $\mathrm{g} / \mathrm{cm}^{3}$ in our balance) and $\rho_{A U}$ and $\rho_{W U}$ the air density in the upper balance chamber housing the built-in weights during measurements of $0_{A}$ and $0_{W}$ respectively. As previously pointed out, there is usually no significant difference between the air density in the upper and lower balance chambers, so, except for the most critical work separate sensors in both of these two chambers are unnecessary. $\rho_{A U}$ and $\rho_{W U}$ are calculated by eq (4) from observations of temperature, barometric pressure and relative humidity recorded during observations for $0_{A}$ and $0_{W}$ respectively.

\section{Data Reduction and Measurements on Silicon}

\subsection{Calculations of Density}

In the Introduction it was stated that the unknown density was obtained from a simultaneous solution of

$$
\begin{aligned}
& \bar{W}_{A}=g\left(M_{X}-\rho_{A} V_{X}\right) \\
& \bar{W}_{W}=g\left(M_{X}-\rho_{W} V_{X}\right)
\end{aligned}
$$

This presupposes that $V_{X}$ has the same value in both the air and water cycles of the experiment, which occurs only when the sample has the same temperature in both cycles. We are ordinarily interested in sample density at some temperature, $T_{N}$, which is not perfectly achieved in either cycle. The simultaneous pair for these general conditions is properly written

$$
\begin{aligned}
& \bar{W}_{A}=g M_{X}-g \rho_{A} V_{T_{N}}\left[1-K\left(T_{N}-t_{A}\right)\right] \\
& \bar{W}_{W}=g M_{X}-g \rho_{W} V_{T_{N}}\left[1-K\left(T_{N}-t_{W}\right)\right]
\end{aligned}
$$

where $V_{T_{N}}$ is the volume of the object at the temperature of interest, $K$ the volumetric coefficient of thermal expansion of the object, $T_{A}$ the air temperature in the pan compartment of the balance during observations for $0_{A}$ and $t_{W}$ the temperature of the water during observations for $0_{W}$. We are justified in assuming that the object of interest lies at temperatures $t_{A}$ and $t_{W}$ during the air and water cycles respectively, under which circumstances the solution of the foregoing pair for sample density at temperature $T_{N}$ is given by

$$
\rho_{T_{N}}=\frac{\bar{W}_{A} \rho_{W} Y-\bar{W}_{W} \rho_{A} X}{\bar{W}_{A}-\bar{W}_{W}}
$$

where

$$
\begin{aligned}
& X=1-K\left(T_{N}-t_{A}\right) \\
& Y=1-K\left(T_{N}-t_{W}\right) .
\end{aligned}
$$

During the two cycles of the experiment, numerical values of the parameters tabulated below are recorded. Where instrumental corrections are appropriate they are assumed made.

\section{Air Weighing Cycle}

$0_{A}=$ the balance observation in whatever units it is calibrated as explained in Air Weighing Procedure, (eq (17)).

$t_{A}=$ the temperature in ${ }^{\circ} \mathrm{C}$ in the pan compartment of the balance.

$t_{A U}=$ the temperature in ${ }^{\circ} \mathrm{C}$ of the air in the upper balance compartment.

$H_{A}=$ the relative humidity (in \%) in the pan compartment of the balance.

$H_{A U}=$ the relative humidity (in \%) in the upper chamber of the balance.

$B_{A}=$ the barometric pressure in $\mathrm{mm} \mathrm{Hg}$ in the laboratory.

$\rho_{S}=$ the density of the built-in weights in $\mathrm{g} / \mathrm{cm}^{3}$.

$K=$ the assumed value of the volumetric coefficient of expansion of the object of interest in parts per part per degree C.

NOTE: As previously pointed out, it is usually unnecessary to install separate temperature and humidity sensors in the two balance chambers; thus $t_{A U}$ may be assumed equal to $T_{A}$ and $H_{A U}$ to $H_{A}$.

\section{Water Weighing Cycle}

$0_{W}=$ the balance observation in units consistent with those in which $0_{A}$ are measured as explained in the Water Weighing Procedure (eq (18)).

$t_{W}=$ the water temperature in ${ }^{\circ} \mathrm{C}$.

$t=$ the interval, in days, since boiling water for degassing.

$B_{W}=$ the barometric pressure in $\mathrm{mm} \mathrm{Hg}$ in the laboratory.

$I=$ the depth of sample immersion in centimeters.

$C=$ compressibility of water $\left(47.7 \times 10^{-6} / \mathrm{atm}\right)$. 
$t_{W U}=$ upper balance chamber air temperature in ${ }^{\circ} \mathrm{C}$. May be observed on thermometer in pan compartment as previously explained.

$H_{W U}=$ upper balance chamber humidity (in \%). May be observed on sensor in lower chamber as previously explained.

$T_{N}=$ the temperature $\left(\right.$ in ${ }^{\circ} \mathrm{C}$ ) at which the sample density is to be determined.

One method of reducing this data to a value of $\rho_{T_{N}}$ is shown in the scheme graphically presented in figure 7 .

In this calculation format it is assumed that the temperature of the unknown object is $T_{A}$ during the air weighing cycle of the experiment and $t_{W}$ during the water cycle. These assumptions are valid, however the use of an assumed value of $K$ may introduce significant error because the actual value of $K$, for a particular object, may differ greatly from its tabulated value. Errors in calculated values of $\rho_{T_{N}}$ associated with erroneous assumptions as to the value of $K$ may be minimixed by holding the factors $\left(T_{N}-t_{A}\right)$ and $\left(T_{N}\right.$ $-t_{W}$ ) near zero by careful adjustment of both the room temperature (and hence $t_{A}$ ) and the water temperature, $t_{W}$, to the desired temperature, $T_{N}$.

This may be impractical since room temperature adjustment is rarely very precise. An alternative approach is to perform the air weighing cycle first at any temperature, $t_{A}$, at the conclusion of which the water cycle is performed with the (relatively easily adjusted) water temperature, $t_{W}$, adjusted to within close tolerance of the previously used $t_{A}$. The value of $T_{N}$ used in the calculation would then be arbitrarily assigned a value of $T_{1}$, midway between $t_{A}$ and $t_{W}$, in which case the density at $T_{1}$ is determined. If $T_{1}$ is too far removed from $T_{N}$, this procedure may be repeated at $T_{2}$ (on the opposite side of $T_{N}$ from $T_{1}$ ) and $\rho_{T N}$ obtained by interpolation between $\rho_{T 1}$ and $\rho_{T 2}$.

A third approach is to make several weighings both

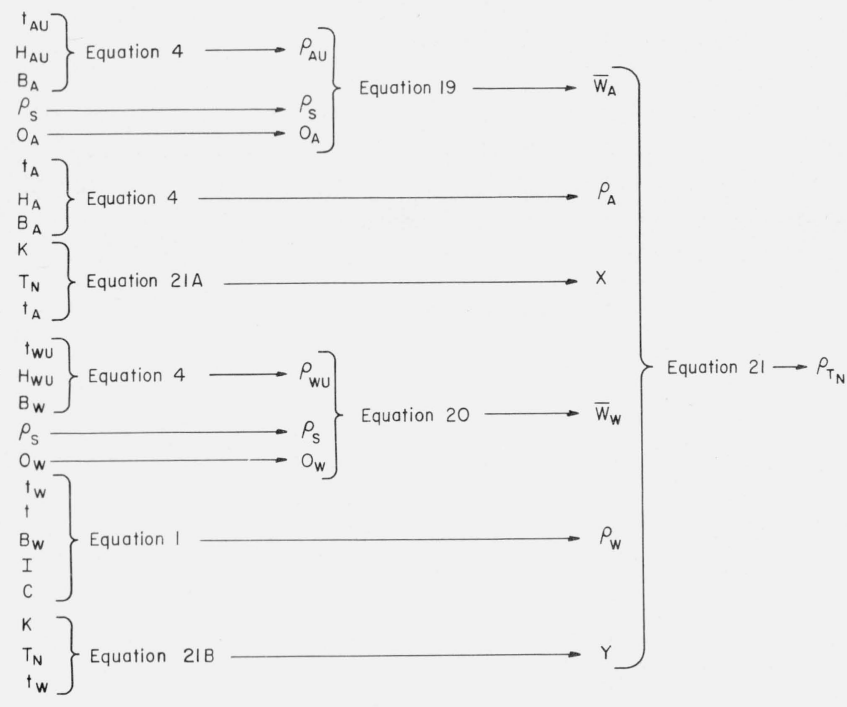

FIGURE 7. Outline of calculations for density. in air and water at various temperatures and form a least squares solution for $M_{X}, V_{T_{N}}$, and $K$, from which $\rho_{T_{N}}=\left(\frac{M_{X}}{V_{T_{N}}}\right)$ may be calculated, and by virtue of knowledge of $K$ the density at any other temperature may be inferred.

\subsection{Observations on Silicon}

As stated in the introduction, one of the objectives of these studies was to determine how much variability in density was to be expected in crystals of silicon weighing 15 to $20 \mathrm{~g}$ cut from the same boule. Experiments (unreported) performed several years age indicated that differences of 20 to 30 parts per million could occur. Recently, two manufacturers submitted samples to NBS for evaluation, which, they felt would prove to be far more homogeneous than the samples previously examined. One boule was grown in a vacuum and one in argon. Three crystals were cut from widely dispersed positions in each boule and each of the six samples were hydrostatically weighed four times using the procedures described in the foregoing sections.

The raw data were reduced as shown in figure 7 to density values presented in table 1 and figure 8 . The average value of the crystals cut from the vacuum grown boule was $2.328982_{4} \mathrm{~g}$ per cubic centimeter with the three crystals spread (in density) over a range of about $2 \mathrm{ppm}$. For the argon-grown boule the average was $2.329022_{1} \mathrm{~g}$ per cubic centimeter and about the same range in values.

TABLE 1

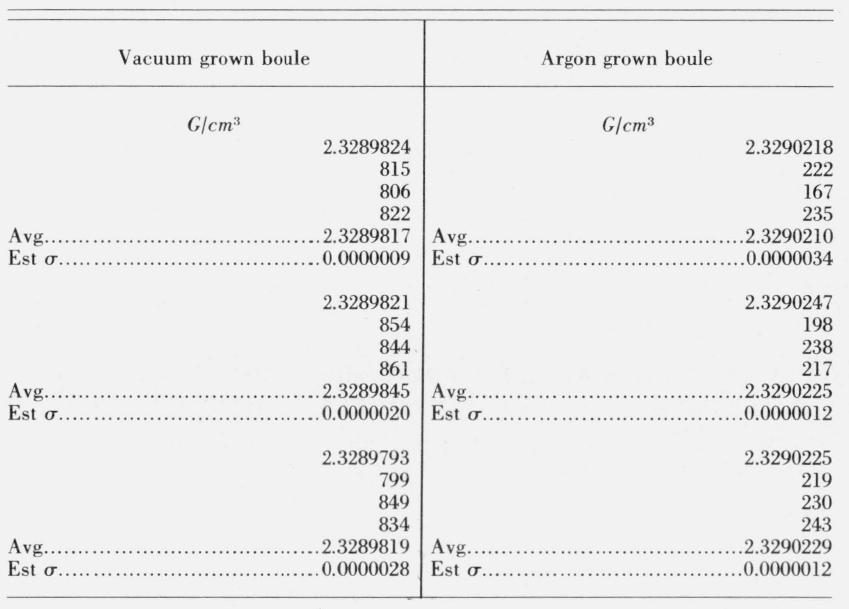

\section{Operating Hints}

During these experiments we have found several techniques which will make the hydrostatic operation much more convenient. The water temperature must be measured with great care inasmuch as this is the parameter responsible for major changes in water 


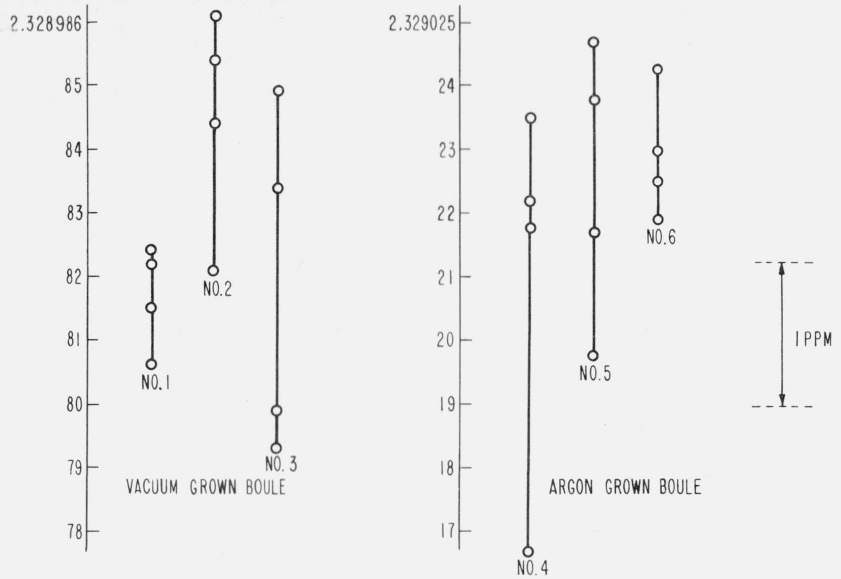

Figure 8. This is a plot of all data taken on all six crystals, from which we may conclude that it is unlikely that the crystals cut from the same boule differ in density by much more than a very few parts per million.

density. We use a platinum resistance thermometer, capsule type, placed at the same horizontal level and close by the sample being measured. This thermometer works into a Mueller Bridge with a wall galvanometer to indicate bridge unbalance. Good practice calls for a reversing switch in the battery line which we habitually use in the measurement. This is not a convenient measurement. In addition to the P.R.T. we also have a high sensitivity electronic thermometer which lacks the accuracy and stability of the P.R.T. but has the virtue of being direct reading to about $0.001{ }^{\circ} \mathrm{C}$. This thermometer is primarily used to indicate when measurements should commence after a load change.

We have a thermal and (therefore) density gradient in the water in our hydrostatic chamber. When samples are removed from the pan and replaced by other samples, turbulent water motion exists thereafter for several minutes (sometimes 20 to $25 \mathrm{~min}$ when the process is carelessly performed). The electric thermometer shows rapid temperature changes of as much as $0.01{ }^{\circ} \mathrm{C}$, up and down, while turbulence exists. When turbulence has subsided, as indicated by a stable temperature, the measurements are commenced.

We habitually keep the ends of the tongs and tweezers used to change the immersed pan loads submerged in the hydrostatic fluid. Although this undoubtedly provides a path for ingress or egress of heat, it has the overriding virtue of guarding against bubbles of air being transferred from the tools to the samples under observation.

Our hydrostatic weighing water is always maintained at a temperature slightly below room temperature. The room temperature may vary as much as $1 / 2$ to $1{ }^{\circ} \mathrm{C}$ during a series of measurements. Water temperature is adjusted to about a degree below the lowest expected room temperature, although we have found that satisfactory measurements can be made with the water as low as 7 or $8 \mathrm{deg}$ below room. If the water temperature is allowed to climb much more than $1 \mathrm{deg}$ above ambient, measurements frequently become erratic. This is usually accompanied by a slight fogging of the glass tube penetrating the water surface, so we assume the upset in measurements is caused by condensation of moisture on the suspension chain within the tube.

When hydrostatic tests are called for upon samples which are too small to fit upon the immersed pan loading device we have found it convenient to use an auxiliary pan (for example, a thin disk of fused silica) which will fit on the unloading device. We then hydrostatically weigh this disk and calculate a value of $\bar{W}_{W}$ for it and then place the sample on the disk and determine a value of $\bar{W}_{W}$ applying to both, followed by a second value of $\bar{W}_{W}$ applying only to the disk. From these measurements it is possible to calculate a value of $\bar{W}_{W}$ applying only to the sample. The reproducibility in a measurement of this type is usually not very good, so such processes must frequently be repeated.

Knife edge balances perform surprisingly well while resting on surfaces which are not perfectly stable (such as a laboratory table with wooden legs). When such balances are used in hydrostatic weighing, vibrations become critical because the surface of the water in the vicinity of the meniscus must be quiet. We have found that looking at the reflection of a brightly lighted object from the underside of the water surface (that is, looking upward through the water at the surface) is a very simple and sensitive test of adequacy of stability. If the reflection of the object is motionless to the unaided eye the vibration level is sufficiently low. If the reflection is unstable, experimental reproducibility will be degraded. This test indicates which doors may not be opened or closed during an experiment or where people may not walk. We believe, without proof, that stability of the hydrostatic weighing chamber is most important, and that vibrations transmitted down the hydrostatic suspension from the balance to the water are somewhat less significant. A slight tap on the hydrostatic weighing chamber will set the water surface in motion, while a similar tap on the balance will hardly be detected.

We have received generous assistance from several people, both inside and outside NBS. Daniel P. Johnson provided guidance on theoretical problems. Richard Deslattes has consulted on a continuing basis and made careful $x$-ray measurements to insure that we experimented on truly state-of-the-art silicon. These remarkably fine crystals were provided by the Dow Corning Corporation and the Texas Instrument Corporation. M. R. Meyerson and J. Kruger consulted on metallurgical problems associated with the suspension wire experiments and Mrs. Theodora S. Welsko made many many fine metallurgical micrographs. Dr. Brenner of the Sigmund Cohn Corporation furnished ideas and experimental samples to aid in the wire experiments. The section on Air Density was prepared with detailed 
guidance from Arnold Wexler, and that on Water Density from John Taylor. Geraldine Hailes and David Wooten assisted in automation of the data reduction and Robert Raybold and Hsien $\mathrm{Ku}$ gave guidance in experimental design and statistical analysis. The frequency of our references to Ivars Henins indicates the extent to which we borrowed from his work. H. S. Peiser assisted with manuscript preparation. His interest in density measurements as a tool for his own field (crystallography) has been a driving force in this and related work for many years. The authors are particularly indebted to Paul Pontius and A. G. $\mathrm{McNish}$ for many ideas and continuing encouragement and support.

\section{References}

[1] H. A. Bowman, R. M. Schoonover, W. H. Gallagher, 14.8-4-65, Proc. ISA - Los Angeles (1966).

[2] I. Henins, J. Res. NBS 68A (Phys. and Chem.), No. 5, 529-533 (1964).

[3] H. A. Bowman, R. M. Schoonover, J. Res. NBS 69C (Engr. and Instr.) No. 3, 217-223 (1965).

[4] H. A. Bowman, H. E. Almer, J. Res. NBS 67C (Engr. and Instr.) No. 3, 227-235 (1963).

[5] M. Thiessen, K. Schell, H. Disselhorst, Wiss. Abh. Phys.-techn. Reichsanst 3, 1-70 (1900).

[6] P. Chappuis, Trav. mem. BIPM 13, D1-D40 (1907).

[7] L. W. Tilton, J. K. Taylor, J. Res. NBS 18, 205 (1937) R.P. 971.

[8] C. E. Guillaume, Trav. mem. BIPM 14, Al-A276 (1910).

[9] P. Chappuis, Trav. mem. BIPM 14, B1-B163 (1910).

[10] J. M. deLepinay, H. Buisson, J. R. Benoit, Trav. mem. BIPM 14, C1-C127 (1910).

[11] I. Friedman, A. C. Redfield, B. Schoen, J. Harris, Rev. Geo. 2, 177-224 (1964).

[12] A. C. Redfield, private communication.

[13] M. Woh and H. C. Urey, J. Chem. Phys. 3, 411-414 (1935).

[14] Christiansen, Crabtree, Loby, Nature 135, 870 (1935).

[15] F. Steckel, S. Szapirs, Trans. Faraday Soc. 59, p. 333 (1963).

[16] Dorsey, Properties of Ordinary Water Substance (Reinhold, New York, 1940).

[17] P. Chappuis, Trav. mem. BIPM 14, D1-D163 (1910).

[18] A. Wexler, NBS Circ. 586 (1957).

[19] A. Wexler, W. G. Brombacher, NBS Circ. 512 (1951).

[20] Harrison, L. P1, pp. 15-16, Humidity and Moisture, 3, edited by Wexler, A. and Wildhack, W. (Reinhold, New York, 1965).

[21] NBS Circular 564, table 2A, p. 25 (1955).

[22] Goff, J. A. and Gratch, S., Smithsonian Meteorological Tables, 6th edition, Smithsonian Institution, Washington, D.C. (1958).

[23] Almer, H. E., Bowman, H. A., Jensen, M. W., Macurdy, L. B., Peiser, H. S. Wasko, B., J. Res. NBS 68C (Engr. and Instr.), No. 3, 141-154 (1964).

\section{Appendix. The Calibration of Built-in Weights of Direct Reading Balances}

\section{W. Jones}

A convenient procedure for obtaining an estimate of the adjustment errors of built-in weights in directreading balances is to load the pan with standard weights, and to ascribe failure of the balance to indicate this known load correctly to adjustment error. This procedure may be carried out for each dial position, except zero, for each of the dials controlling the built-in weights. Thus, nine loads from 1 to $9 \mathrm{~g}$ in $1 \mathrm{~g}$ steps serve to calibrate the $1 \mathrm{~g}$ dial, or 0.1 to 0.9 in $0.1 \mathrm{~g}$ steps for the $0.1 \mathrm{~g}$ dial.
Good practice demands that each comparison of a "known" pan load to an "unknown" built-in load be carried out by the double substitution weighing procedure. With one exception, the double substitution format described in the paragraph "Air Weighing Procedure" of section 6 of the paper is quite adequate for the calibration experiment. In the density measurement procedure described in the basic paper the equation for $0_{A}$, requires corrected values of the dial readings. Inasmuch as this experiment is directed toward the determination of the dial corrections we must alter the meaning of $D_{t 1}$ and $D_{t 2}$ from corrected dial readings to their raw values.

In the calibration mode the value of $0_{A}$ is properly written

$$
0_{A}=D_{2}-D_{1}+\frac{G\left(s_{2}-s_{1}+s_{3}-s_{4}\right)}{\left(s_{3}-s_{2}+s_{4}-s_{5}\right)}
$$

and since in an air weighing experiment of this type, $D_{1}$ is always zero, the expression becomes

$$
0_{A}=D_{2}+\frac{G\left(s_{2}-s_{1}+s_{3}-s_{4}\right)}{\left(s_{3}-s_{2}+s_{4}-s_{5}\right)}
$$

where $D_{2}$ is the dial position under calibration, $G$, the values of the sensitivity weight and $s_{1}$ to $s_{5}$ the screen readings observed during each of the five steps of the double substitution experiment. With a value of $0_{A}$, we may calculate $C$, the correction to $D_{2}$, thus:

$$
C=\frac{\left(M_{S}+C_{S}\right)\left(1-\frac{\rho_{S}}{\rho_{A}}\right)}{1-\frac{\rho_{B W}}{\rho_{A}}}-0_{A}
$$

where $M_{S}, C_{S}$ and $\rho_{S}$ are the mass, correction and density, respectively, of the standard weight on the pan, $\rho_{B W}$ the density of the built-in weights and $\rho_{A}$ the air density in the balance case at the time of the experiment.

Statements concerning the accuracy of measured values of loads obtained on direct reading balances calibrated by the foregoing procedure should be made with extreme caution. If the standard deviation of a single measurement on such a balance in the double substitution mode is $\sigma$, then our knowledge of the corrections to the built-in weights is limited to about $\sigma$ if the calibration operation was performed once. When a single measurement on an unknown load is made on this balance the variability of the balance comes into play a second time, so our knowledge of the unknown load is limited to a bit less than $2 \sigma$, and for a 99 percent confidence interval this figure should be multiplied by 3 . Additionally, it is necessary to add 1 or 2 $\mu \mathrm{g}$ as our estimate of the uncertainty of the corrections to the standard weights used in the calibration.

(Paper 71C3-254) 